\section{Descuento de flujos de fondos, inflación, tipo de cambio y COVID-19: un modelo aplicado en el mercado argentino}

Cash flow discount, inflation, exchange rate and

COVID-19: a model applied in argentinian market

\section{Gastón S. Milanesi}

\section{RESUMEN}

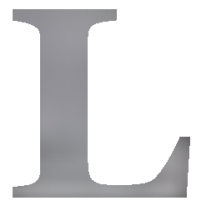

a actual crisis sanitaria se traduce en un cisne negro que impactó en todas las economías. A diferencia de otras crisis cuyo impacto fue general, esta afectó determinados segmentos de negocios. En particular aquellos con mercados enfocados al transporte, ocio y consumo discrecional, con uso intensivo de factor humano para operaciones y significativo apalancamiento financiero aplicado a significativos stocks de capital. En esa línea los modelos de valuación de empresa, más que nunca, deben apoyarse en los fundamentos de valor y los riesgos macroeconómicos. Argentina, como mercado emergente, suma la dificultad del importante proceso inflacionario. El trabajo propone un modelo de valuación donde los riesgos propios y fundamentos de valor son incorporados en los flujos proyectados y los riesgos sistémicos son contenidos en las tasas. El modelo plantea la valuación en dos monedas, curso legal y dólares EE.UU., en el marco de las teorías de paridad de tipos de interés y poder adquisitivo. El valor de la firma será en función del crecimiento real de los ingresos y la vuelta a niveles de márgenes operativos prepandemia, variables analizadas con método de simulación Monte Carlo. El funcionamiento del modelo se ilustra mediante la valuación de las empresas cotizantes en el mercado local al 12 de junio del 2020, exponiendo una destrucción de valor intrínseco medido en dólares del $38 \%$.

Palabras clave: COVID-19; descuento de flujos de fondos; tipo de cambio inflación; valor intrínseco.

\section{ABSTRACT}

The current health crisis translates into a black swan that impacted all economies. Unlike other crises whose impact was general, this
Gastón S. Milanesi milanesi@uns.edu.ar

Departamento Ciencias de la Administración, Universidad Nacional del Sur

CEA, Centro de Estudios en Administración, Universidad Nacional del Sur

CEPAF Centro de Estudios para Análisis Financiero,

Facultad de Ciencias Económicas, Universidad de Buenos Aires

Universidad Tecnológica Nacional, Facultad Regional Bahía Blanca, Departamento de Licenciatura en Organización Industrial ARGENTINA

\section{COMO CITAR ESTE ARTÍCULO} Gastón Milanesi, S. (2020). Descuento de flujos de fondos, inflación, tipo de cambio y COVID-19: un modelo aplicado en el mercado argentino. Revista de la Facultad de Ciencias Económicas, 25(2), 33-49. http://dx.doi.org/10.30972/rfce.2524564

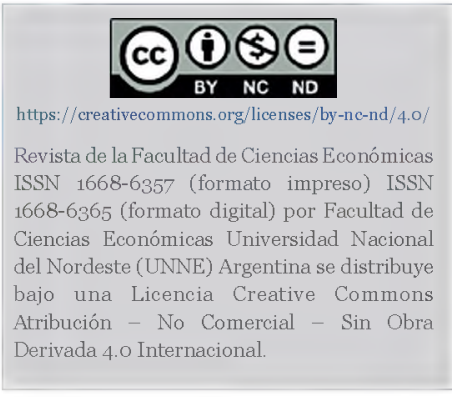


one affected certain business segments. Particularly those with markets focused on transportation, leisure and discretionary consumption, with intensive use of the human factor for operations and significant financial leverage applied to significant capital stocks. Along these lines, company valuation models, more than ever, must be based on value fundamentals and macroeconomic risks. Argentina, as an emerging market, adds to the difficulty of the important inflationary process. The work proposes a valuation model where the own risks and value foundations are incorporated in the projected flows and the systemic risks are contained in the rates. The model proposes the valuation in two currencies, legal tender and US dollars, within the framework of theories of parity of interest rates and purchasing power. The firm's value will be a function of real revenue growth and the return to pre-pandemic operating margin levels, variables analyzed with the Monte Carlo simulation method. The operation of the model is illustrated by the valuation of listed companies in the local market as of June 12, 2020, exposing an destruction of intrinsic value measured in dollars of $38 \%$.

Keywords: COVID-19; cash flow discount; exchange rate; inflation; intrinsic value.

\section{INTRODUCCIÓN}

Los procesos de valuación de empresas en marcha es el resultado de una tarea intelectual de toma de conocimiento de variables como el contexto macroeconómico, estructura del sector, posicionamiento en el mercado y estrategias, se transforma en parámetros cuantitativos para la toma de decisiones. En otras palabras, el valor es función directa del conocimiento del estado actual y prospectivo correspondiente a las variables que definen el valor. El valor teórico o intrínseco ${ }^{1}$ surge de la actualización a una tasa ajustada por riesgo de la corriente proyectada de beneficios esperados. En este caso el modelo de mayor uso entre los practicantes es el descuento de flujo de fondos (DFF), (Copeland, T., Koller, K., Murrin, J., 2000); (Ruback, 2002); (Damodaran A., 2006); (Damodaran, A., 2009) (Booth, 2007); (Koller, T., Goedhart, M., Wessels, D., 2010); (Fernández, 2012); (Damodaran A., 2012); (Milanesi, 2017)². El valor obtenido representa el supuesto valor

\footnotetext{
${ }^{1}$ El valor representa las expectativas del evaluador (comprador/vendedor), en el caso de activos financieros, en mercado eficientes, perfectos y con expectativas homogéneas, las partes coinciden en la estimación de valor, y por lo general este representa el precio. En el caso de activos reales (empresas o activos fijos), donde las expectativas son heterogéneas, las partes no coinciden en su apreciación de valor, se da un proceso no instantáneo de negociación, donde el precio surge del acuerdo de las partes y este puede distar del valor inicial para el comprador/vendedor. Por ello, el precio refleja expectativas pasadas, mientras que el valor refleja las expectativas futuras del agente.

${ }^{2}$ Respecto del costo del capital los tres métodos empleados: a) Costo Capital Promedio Ponderado (ccpp, wacc), b) Flujos de fondos a capital, c) Valor Presente Ajustado (APV). En el presente trabajo se adoptará la técnica del ccpp. Existen otros modelos como las Ganancias Residuales (Residual Income) y Valor Económico Añadido (Economic Value Added). Son otras alternativas para presentar el mismo valor obtenido por el modelo de descuento de flujos de fondos (Fernández, 2014).
} 
intrínseco de la firma en marcha desde la perspectiva del inversor diversificador de riqueza en carteras. ${ }^{3}$

En condiciones normales la ecuación de valor intrínseco de los activos de la firma es:

$$
V_{t=0}=\sum_{t=1}^{\mathrm{n}} \frac{E\left(F F_{t}\right)}{\left(1+c c p p_{t}\right)^{t}}=\frac{E\left(F F_{t, 1}\right)}{\left(1+c c p p_{t+1}\right)^{t+1}}+\frac{E\left(F F_{t+2}\right)}{\left(1+c c p p_{t+2}\right)^{t+2}}+\ldots+\frac{\frac{E\left(F F_{n}\right)}{\left(c c p p_{n-} g_{n}\right)}}{\left(1+c c p p_{n}\right)^{n}}
$$

Donde $\mathrm{V}_{\mathrm{t}=\mathrm{o}}$ representa el valor intrínseco de los activos de la firma, $\mathrm{E}(\mathrm{FF})$ el flujo de fondos esperados, el cual es la esperanza matemática de los flujos de fondos proyectados para cada periodo y ccpp es el costo del capital promedio ponderado para cada periodo. La ecuación anterior se divide en dos horizontes temporales; el primero conocido como horizonte explícito de proyección, donde se proyectan específicamente el comportamiento cambiante los conductores de valor (value driver), atendiendo al contexto macro, ventajas competitivas de la firma y estrategia de posicionamiento. El segundo se conoce como valor terminal donde se supone que la firma ingresa en un comportamiento estable (steady state).

En contextos de alta incertidumbre como los que atraviesa la humanidad frente a la pandemia desatada por el COVID-19, es menester establecer las pautas de valuación de activos. Estas nunca deben abandonar los fundamentos por los cuales se generan valor, deben complementar herramientas y métricas que incorporen las nuevas exposiciones a riesgos.

En este breve artículo se presentará de manera operativa, referenciando los modelos y ecuaciones para los interesados en profundizar su estudio. Simplemente se expondrá un sencillo marco de valuación adaptado al mercado emergente local. Este modelo reconoce su base en los drivers de valor incorporando teorías de paridad, valuación en moneda dura ${ }^{4}$ y simulación Monte Carlo para el tratamiento del riesgo y convergencia de márgenes. El caso analizado será el agregado de los drivers correspondientes a las firmas cotizantes, suponiendo que el mercado local es una empresa, donde los fundamentos de riqueza explican su valor.

La estructura del artículo es la siguiente: en la primera parte se expondrán los valores correspondientes a la evolución de los mercados de equity internacionales y por sector, con el fin de exponer los desempeños en febrero, marzo y mayo (prepandemia y salida de cuarentena. En estos cuadros se podrá apreciar los sectores más afectados y la recuperación. Utilizando diagramas será expuesto el modelo tradicional y sus ajustes para valuar flujos, construir tasas, obtener valores intrínsecos y la estructura del riesgo. Para ello será utilizado el sector cotizante agregado en el mercado local, comparando valor previo y durante esta pandemia.

\footnotetext{
${ }^{3}$ El valor obtenido surge de actualizar flujos esperados a una tasa de costo del capital que refleja el riesgo sistémico, aquella fracción de la varianza no evitable mediante diversificación eficiente. La tasa es estimada por modelos basados en el CAPM (Capital Asset Princing Model), y no refleja el riesgo de las diferentes estrategias o flexibilidad estratégica de la firma. Para ello es menester emplear un enfoque de opciones reales (Milanesi, 2013); (Milanesi, 2018)

${ }^{4}$ En virtud al actual proceso inflacionario, es menester trabajar con monedas duras como el dólar estadounidense pues la moneda doméstica si bien cumple con la función de unidad de medida, no cumple satisfactoriamente las funciones de reserva de valor y poder irrestricto de cancelación de obligaciones, sobre todo cuando la transacción involucra intercambio de flujos financieros a mediano y largo plazo en activos reales. (Milanesi, G., Weins, G., Pequeño, D., 2020).
} 


\section{2- LOS CONDUCTORES DE VALOR Y EL COVID-19}

Existe mucha incertidumbre alrededor de las economías, el valor de los activos financieros y en particular de los activos reales, situación que atenta contra el uso de los modelos de valuación. No obstante, son estos momentos donde estos modelos cobran relevancia, poniendo énfasis en los conductores o generadores de valor, con ajuste en los precios del riesgo producto de la pandemia. La aseveración precedente surge de analizar la evolución global de los principales índices por región, país y por sector de la economía, conforme se expone en las siguientes tablas:

Tabla 1: Evolución por región de los principales índices (febrero-marzo-mayo 2020).

\begin{tabular}{|c|c|c|c|c|c|c|c|c|}
\hline Región & Indice & País & febrero & marzo & mayo & $\begin{array}{c}\text { febrero- } \\
\text { marzo }\end{array}$ & $\begin{array}{c}\text { febrero- } \\
\text { mayo }\end{array}$ & $\begin{array}{c}\text { marzo- } \\
\text { mayo }\end{array}$ \\
\hline \multirow{6}{*}{ América } & S\&P500 & US & 3214 & 2305 & 2831 & $-28,28 \%$ & $-11,92 \%$ & $22,82 \%$ \\
\hline & NASDAQ 100 & US & 9624 & 6994 & 8718 & $-27,33 \%$ & $-9,41 \%$ & $24,65 \%$ \\
\hline & TSX & Canada & 17848 & 11852 & 14620 & $-33,59 \%$ & $-18,09 \%$ & $23,35 \%$ \\
\hline & IPC MEX & México & 45000 & 34270 & 36470 & $-23,84 \%$ & $-18,96 \%$ & $6,42 \%$ \\
\hline & IBOVESPA & Brazil & 114381 & 67069 & 80506 & $-41,36 \%$ & $-29,62 \%$ & $20,03 \%$ \\
\hline & MERVAL & Argentina & 41404 & 22857 & 41319 & $-44,80 \%$ & $-0,21 \%$ & $80,77 \%$ \\
\hline \multirow{4}{*}{ Europa } & FTSE 100 & UK & 7409 & 5191 & 5763 & $-29,94 \%$ & $-22,22 \%$ & $11,02 \%$ \\
\hline & $\mathrm{DAX}$ & Alemania & 13744 & 8929 & 10862 & $-35,03 \%$ & $-20,97 \%$ & $21,65 \%$ \\
\hline & $\mathrm{CAC}_{40}$ & Francia & 6069 & 4131 & 4559 & $-31,93 \%$ & $-24,88 \%$ & $10,36 \%$ \\
\hline & S\&P EU $35^{\circ}$ & Europa & 1731 & 1181 & 1351 & $-31,77 \%$ & $-21,95 \%$ & $14,39 \%$ \\
\hline \multirow{4}{*}{ Asia } & NIKKEI 225 & Japón & 23688 & 16553 & 19619 & $-30,12 \%$ & $-17,18 \%$ & $18,52 \%$ \\
\hline & SHANGHAI $5^{\circ}$ & China & 2895 & 2628 & 2862 & $-9,22 \%$ & $-1,14 \%$ & $8,90 \%$ \\
\hline & HANG SENG & Hong Kong & 27816 & 22805 & 24644 & $-18,01 \%$ & $-11,40 \%$ & $8,06 \%$ \\
\hline & SENSEX & India & 41258 & 29916 & 33718 & $-27,49 \%$ & $-18,28 \%$ & $12,71 \%$ \\
\hline Australia \& & ASX 200 & Australia & 7133 & 4825 & 5246 & $-32,36 \%$ & $-26,45 \%$ & $8,73 \%$ \\
\hline $\mathrm{NZ}$ & $\mathrm{NZX}_{5} \mathrm{O}$ & Nueva Zelanda & 11835 & 9202 & 10449 & $-22,25 \%$ & $-11,71 \%$ & $13,55 \%$ \\
\hline \multirow{2}{*}{ África } & FTSW/JSE TOP 40 & Sud África & 52050 & 36302 & 46188 & $-30,26 \%$ & $-11,26 \%$ & $27,23 \%$ \\
\hline & NSE - All Share & Nigeria & 27756 & 22198 & 23021 & $-20,02 \%$ & $-17,06 \%$ & $3,71 \%$ \\
\hline
\end{tabular}

Fuente: elaboración propia en base a los datos contenidos en el sitio A. Damodaran http://pages.stern.nyu.edu/ adamodar/

La tabla expone el impacto del COVID-19 en los principales índices, tomando dos extremos como son los periodos febrero-marzo; febrero-mayo y un intermedio marzo-mayo. Cabe destacar que los índices pueden tomarse como indicador de la evolución de una economía, a través de los precios de los activos financieros ${ }^{5}$, representativos de las expectativas sobre la evolución futura del desempeño y estructura financiera de empresas como gobiernos. El primer intervalo de tiempo muestra una profunda caída en el valor de los activos, producto de

\footnotetext{
${ }^{5}$ Estos presentan una reacción elástica o de mayor velocidad frente a las variaciones en las expectativas de los agentes, si se los compara con los activos reales.
} 
la significativa incertidumbre generada por la pandemia. Los valores negativos de la segunda columna (febrero-mayo) son significativamente inferiores. La explicación aparente se encuentra contenida en la tercera columna, donde todas las economías ingresaron en una fase de recuperación en lo que respecta al valor de los activos financieros. La siguiente tabla analiza el comportamiento de los sectores de la economía a nivel mundial de un conjunto de 36.419 firmas, en los periodos de referencia. A diferencia de otras crisis donde el impacto fue global, en este caso afectó a determinados modelos de negocios o segmentos de la economía.

Tabla 2: Creación - destrucción de valor por sector (febrero-marzo-mayo 2020).

\begin{tabular}{|c|c|c|c|c|c|c|c|c|c|c|}
\hline \multirow{2}{*}{ Sector } & \multirow{2}{*}{ Empr. } & \multicolumn{6}{|c|}{ Capitalización de mercado en miles de millones de u\$ } & \multicolumn{2}{|r|}{ Variación } & \multirow[b]{2}{*}{ mar a may } \\
\hline & & & febrero & & marzo & & mayo & feb. a mar & feb. a may & \\
\hline $\begin{array}{l}\text { Servicios de } \\
\text { comunicación }\end{array}$ & 1690 & $\$$ & $7.342 .037,00$ & $\$$ & $5.488 .763,00$ & $\$$ & $6.489 .211,00$ & $-25,24 \%$ & $-11,62 \%$ & $18,23 \%$ \\
\hline $\begin{array}{c}\text { Consumo selectivo } \\
\text { no escencial }\end{array}$ & 4930 & $\$$ & $10.148 .621,00$ & $\$$ & $7.059 \cdot 451,00$ & $\$$ & $8.539 \cdot 340,00$ & $-30,44 \%$ & $-15,86 \%$ & $20,96 \%$ \\
\hline $\begin{array}{l}\text { Consumo básico } \\
\text { (alimentos y bebidas) }\end{array}$ & 2300 & $\$$ & $7.116 .299,00$ & $\$$ & $5.701 .278,00$ & $\$$ & $6.476 .403,00$ & $-19,88 \%$ & $-8,99 \%$ & $13,60 \%$ \\
\hline Energía & 1313 & $\$$ & $5.930 .247,00$ & $\$$ & $3.852 .615,00$ & $\$$ & $4.586 .674,00$ & $-35,03 \%$ & $-22,66 \%$ & $19,05 \%$ \\
\hline Financiero & 3853 & $\$$ & $14.919 .880,00$ & $\$$ & $9.915 .070,00$ & $\$$ & $11.045 \cdot 925,00$ & $-33,54 \%$ & $-25,97 \%$ & $11,41 \%$ \\
\hline Salud & 3408 & $\$$ & $8.970 .146,00$ & $\$$ & $6.909 .417,00$ & $\$$ & $8.529 .498,00$ & $-22,97 \%$ & $-4,91 \%$ & $23,45 \%$ \\
\hline Industria & 6546 & $\$$ & $10.112 .845,00$ & $\$$ & $6.894 .349,00$ & $\$$ & $8.021 .543,00$ & $-31,83 \%$ & $-20,68 \%$ & $16,35 \%$ \\
\hline $\begin{array}{l}\text { Tecnología e } \\
\text { Información }\end{array}$ & 4867 & $\$$ & $13.594 .754,00$ & $\$$ & $9.734 .631,00$ & $\$$ & $11.846 .660,00$ & $-28,39 \%$ & $-12,86 \%$ & $21,70 \%$ \\
\hline $\begin{array}{l}\text { Materiales y partes } \\
\text { intermedias }\end{array}$ & 4400 & $\$$ & $4.988 .086,00$ & $\$$ & $3.526 .263,00$ & $\$$ & $4.233 .418,00$ & $-29,31 \%$ & $-15,13 \%$ & $20,05 \%$ \\
\hline Real State & 2298 & $\$$ & $4.040 .386,00$ & $\$$ & $2.699 .214,00$ & $\$$ & $3.143 .627,00$ & $-33,19 \%$ & $-22,19 \%$ & $16,46 \%$ \\
\hline $\begin{array}{l}\text { Utilities (servicio } \\
\text { público) }\end{array}$ & 814 & $\$$ & $3.199 .760,00$ & $\$$ & $2.326 .774,00$ & $\$$ & $2.640 .444,00$ & $-27,28 \%$ & $-17,48 \%$ & $13,48 \%$ \\
\hline Global & 36419 & $\$$ & $90.363 .061,00$ & $\$$ & $64.107 .825,00$ & $\$$ & $75.552 .743,00$ & $-29,06 \%$ & $-16,39 \%$ & $17,85 \%$ \\
\hline
\end{tabular}

Fuente: elaboración propia en base a los datos contenidos en el sitio A. Damodaran http://pages.stern.nyu.edu/ adamodar/ http://pages.stern.nyu.edu/ adamodar/

Se puede decir que existe una aparente racionalidad en como los mercados ajustan el valor de los activos financieros. El mayor desempeño lo han tenido las industrias que aportan soluciones inmediatas a los problemas que trae aparejado el COVID-19 como los sectores de cuidado de la salud, medicamentos, biotecnología, informática, retail electrónico y alimentación. Son modelos de negocios que se ajustan a la dinámica de la pandemia, generando beneficios con bajas inmovilizaciones de capital, y en el estado actual, atienden necesidades prioritarias. En particular en economías desarrolladas uno de los segmentos más castigados por el COVID-19 es el de los servicios financieros; ya que estos tienen colocadas sus carteras de activos (préstamos) en diferentes sectores de la economía que presentan graves inconvenientes como la exploración y refinamiento de petróleo, aerolíneas y servicios turísticos, por ejemplo. No obstante, la flexibi- 
lidad del modelo de negocio hace presumir su recuperación. Otro sector castigado es la energía, en este caso se combinaron factores como la caída de la demanda global, exceso de oferta y un impacto en el precio del barril. ${ }^{6}$

El denominador común para muchos de estos negocios afectados por el COVID-19 es el excesivo financiamiento mediante deuda. El leverage financiero presenta efectos positivos en el financiamiento del crecimiento cuando los mercados evolucionan favorablemente. Ante caídas en los niveles de actividad el impacto negativo en los resultados y expectativas ajusta de manera profunda y asimétrica ${ }^{7}$, en otros términos, beneficios inversos asimétricos a los generados por una posición larga en una opción de compra.

No obstante, la crisis pone en jaque algunos mitos del valor ya que firmas con ratios "seguros", como por ejemplo bajos PER (price earnings ratio) y altos rendimientos de dividendos (dividend yields); de aparente estabilidad dejaron de ser inversiones seguras, productos de su fundamentals de valor: modelos de negocios afectados por baja actividad, alta inversión en activos fijos y significativo leverage. En las siguientes tablas se presentan los sectores con peor y mejor desempeño.

Tabla 3: Los peores segmentos (febrero-marzo-mayo 2020).

\begin{tabular}{|c|c|c|c|c|c|c|c|c|c|c|}
\hline \multirow{2}{*}{ Sector } & \multirow{2}{*}{ Empr. } & \multicolumn{6}{|c|}{ Capitalización de mercado en miles de millones de $u \$$} & \multicolumn{3}{|c|}{ Variación } \\
\hline & & & febrero & & marzo & & mayo & feb. a mar & feb. a may & mar a may \\
\hline Transporte aéreo & 150 & $\$$ & $556.178,00$ & $\$$ & $313.482,00$ & $\$$ & $351.688,00$ & $-43,64 \%$ & $-36,77 \%$ & $12,19 \%$ \\
\hline $\begin{array}{c}\text { Petróleo/Gas (producción y } \\
\text { exploración) }\end{array}$ & 480 & $\$$ & $692.963,00$ & $\$$ & $318.155,00$ & $\$$ & $444.392,00$ & $-54,09 \%$ & $-35,87 \%$ & $39,68 \%$ \\
\hline Aeroespacio y defensa & 212 & $\$$ & $1.217 .844,00$ & $\$$ & $674.146,00$ & $\$$ & $\$ 799.015,00$ & $-44,64 \%$ & $-34,39 \%$ & $18,52 \%$ \\
\hline $\begin{array}{c}\text { Broadcasting (servicios } \\
\text { de difusión) }\end{array}$ & 121 & $\$$ & $164.681,00$ & $\$$ & $93.967,00$ & $\$$ & $108.625,00$ & $-42,94 \%$ & $-34,04 \%$ & $15,60 \%$ \\
\hline Hoteles y esparcimiento & 505 & $\$$ & $715.671,00$ & $\$$ & $386.318,00$ & $\$$ & $483.034,00$ & $-46,02 \%$ & $-32,51 \%$ & $25,04 \%$ \\
\hline Construcción de hogares & 147 & $\$$ & $247.004,00$ & $\$$ & $127.536,00$ & $\$$ & $166.749,00$ & $-48,37 \%$ & $-32,49 \%$ & $30,75 \%$ \\
\hline Reaseguro & 33 & $\$$ & $180.567,00$ & $\$$ & $98.174,00$ & $\$$ & $122.043,00$ & $-45,63 \%$ & $-32,41 \%$ & $24,31 \%$ \\
\hline Comidas mayorista & 118 & $\$$ & $93.191,00$ & $\$$ & $50.097,00$ & $\$$ & $64.748,00$ & $-46,24 \%$ & $-30,52 \%$ & $29,25 \%$ \\
\hline $\begin{array}{l}\text { Petróleo/Gas } \\
\text { (distribución) }\end{array}$ & 186 & $\$$ & $649.295,00$ & $\$$ & $357.879,00$ & $\$$ & $455.453,00$ & $-44,88 \%$ & $-29,85 \%$ & $27,26 \%$ \\
\hline
\end{tabular}

Fuente: elaboración propia en base a los datos contenidos en el sitio A. Damodaran http://pages.stern.nyu.edu/ adamodar/

En síntesis, los conductores de valor son los que justifican la valuación y estos se encuentran íntimamente ligados al modelo de negocio. Empleando metáforas epidemiológicas, el foco de contagio del COVID-19, durante y postpandemia manifiesta sus riesgos en drivers de valor y sectores que se encuentran vinculados:

- Ingresos vinculados a actividades de transporte recreativo o servicios turísticos (Ingresos)

\footnotetext{
${ }^{6}$ A la fecha con caída del 52,3\% para el Brent y $62,5 \%$ WTI respectivamente.

${ }^{7}$ Sería la inversa del perfil de asimetría para el tenedor de una opción de compra (call), potenciando pérdidas, en otras palabras, "el lado oscuro de la deuda" (Damodaran, 2009).
} 
- Ingresos vinculados a producción de bienes para consumo discrecional con posibilidad de diferir compras (Ingresos)

- El uso intensivo del factor humano en operaciones, con alta participación en el costo operativo (Costos de Operación)

- Negocios con importante estructura de costo fijo y poca flexibilidad que resultan en importantes efectos de palanca operativo (Inversiones)

- Negocios con endeudamientos significativos, en especial con bajos ratios de cobertura de intereses, alto efecto palanca financiero (Deuda)

- Empresas en crecimiento con altas necesidades de flujos de efectivo a través del mercado de deuda (Deuda).

El siguiente gráfico muestra el mapa de contagio y riesgos a los que se exponen los sectores.

Gráfico 1: El mapa del contagio y los drivers de valor.

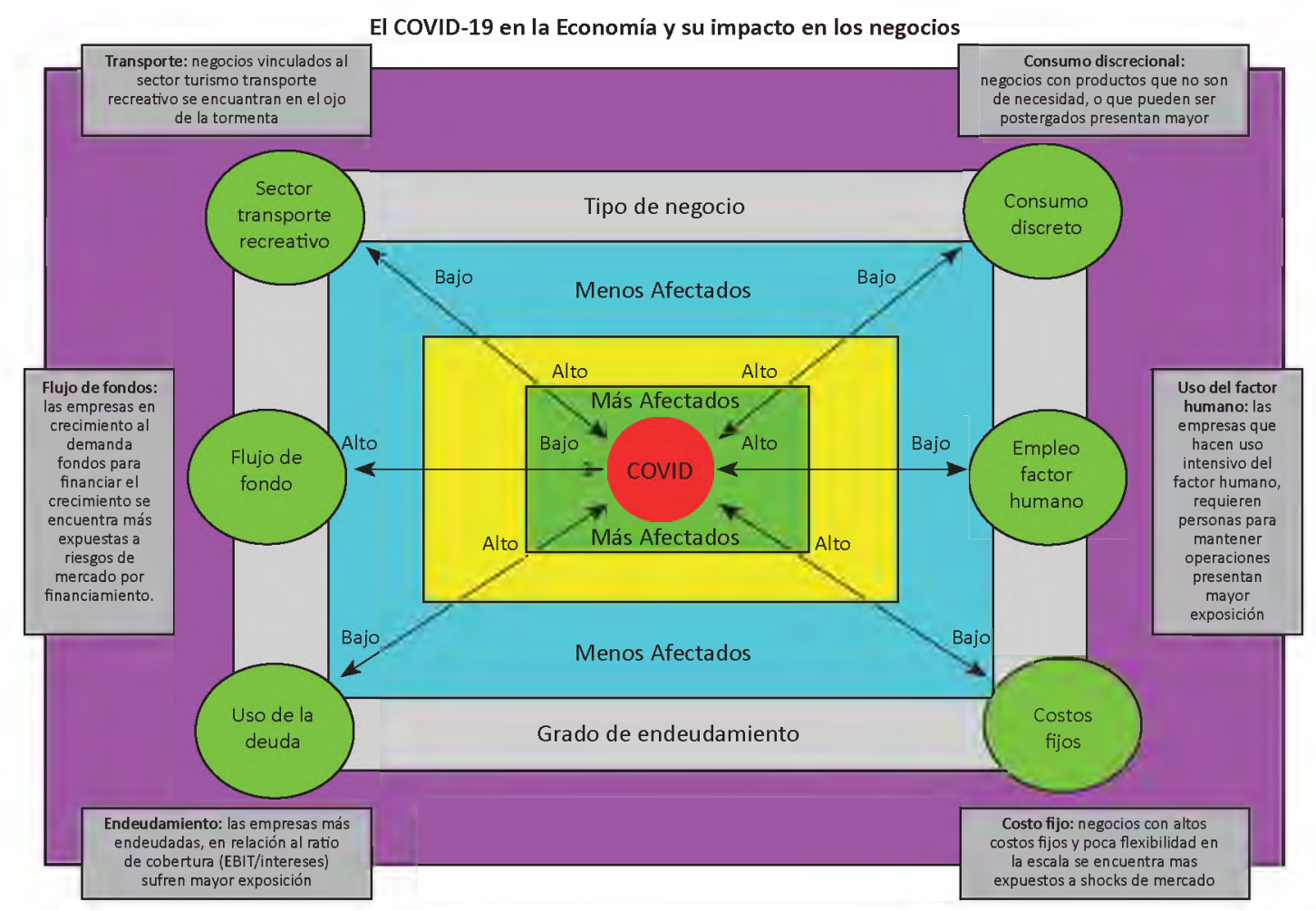

Fuente: elaboración propia. 


\section{3-EL VALOR DE LA FIRMA Y EL COVID-19}

En base a lo expuesto valuar requiere más que nunca prestar atención en el contexto y los fundamentals de valor del negocio. El siguiente gráfico presenta las variables propias del modelo de valuación, en función a la clásica expresión de valor de una empresa en marcha, según el modelo de descuento de flujos de fondos (ecuación 1).

Gráfico 2: El valor de la firma en condiciones normales.

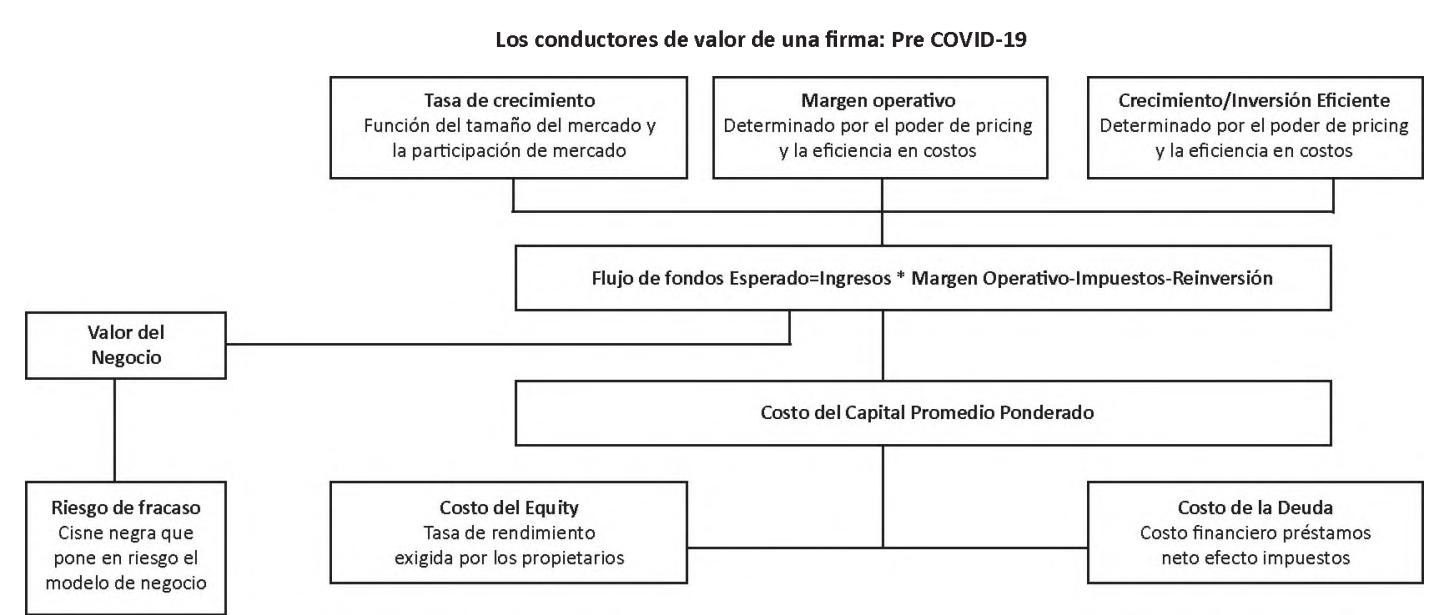

Fuente: elaboración propia.

En un contexto de pandemia y en economías emergentes y altamente volátiles la lógica del valor debe adaptarse al momento especial como al comportamiento esperado en el mediano plazo de la macroeconomía. Por ello, el modelo incorpora el clásico tratamiento de generación de valor (drivers) amplificado con simulación Monte Carlo, el impacto macroeconómico en el mediano y largo plazo sobre el poder adquisitivo de la unidad de medida y determinación del costo del capital en una economía emergente. Esto último en base a las teorías de paridad del poder adquisitivo, tipos de cambios esperados y tipos de interés (Milanesi, 2017). El modelo opera los riesgos propios del mercado e idiosincráticos del sector en los flujos, reservando el ajuste por riesgos sistémicos a la tasa de descuento. En términos de ecuación de descuento de flujos de fondos se tiene:

a) Fhijos de fondos esperados: proyectar inflación local y tasas de crecimiento reales por separado, para obtener flujos de fondos proyectados en moneda doméstica de poder adquisitivo proyectado para cada periodo y convertirlos a dólares estadounidenses futuros.

b) Tasa de costo del capital: en base a la calificación crediticia, evolución de los tipos de interés y riesgo sistémico este se determina en moneda dura, para luego ser convertido a moneda de curso legal. 
En la siguiente ilustración se encuentra expresada la lógica del modelo propuesto:

Gráfico 3: El valor de la firma en condiciones COVID-19.

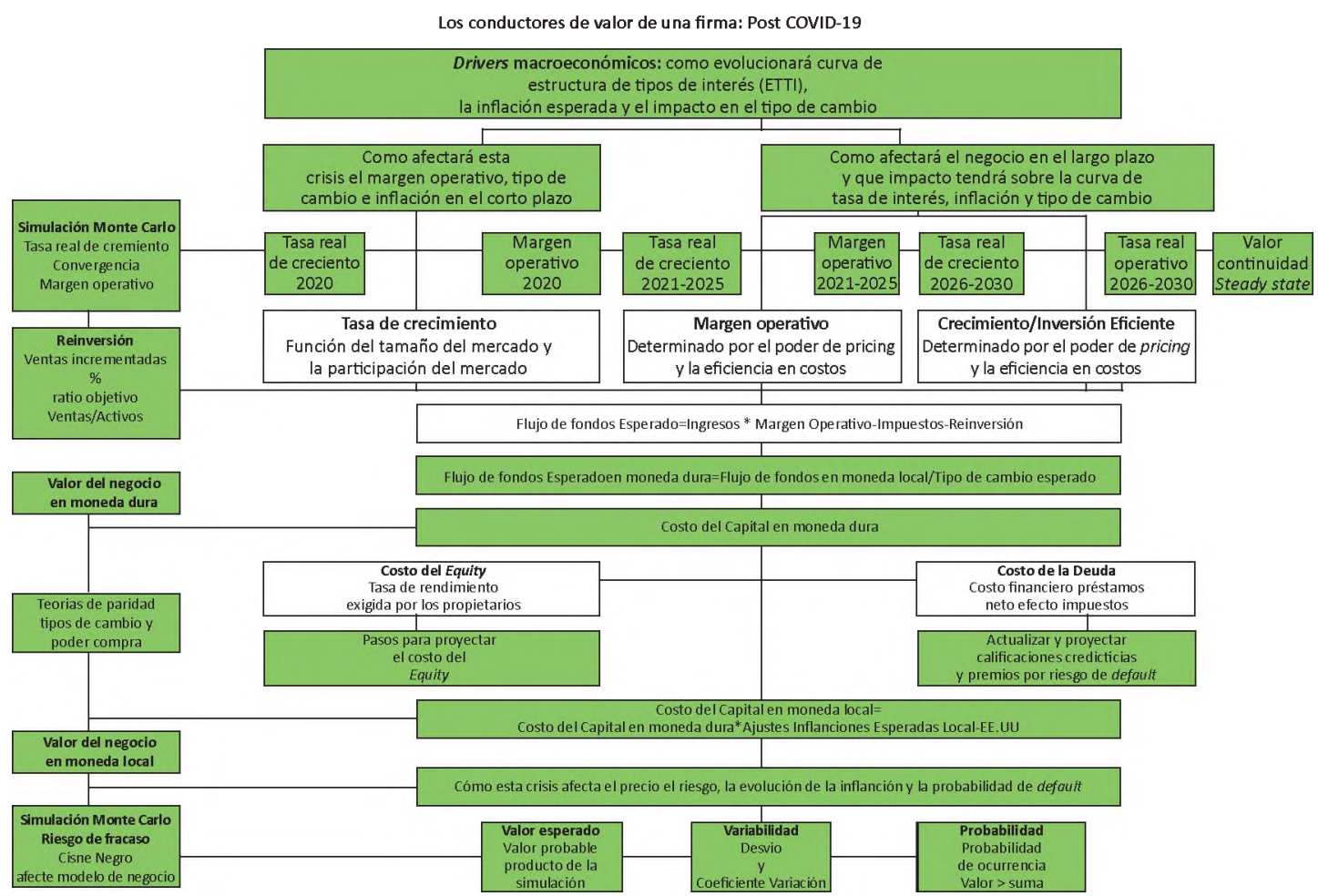

Fuente: elaboración propia.

\section{1- PROYECCIÓN DE MAGNITUDES FINANCIERAS MACROECONÓMICAS}

El modelo comienza con la proyección de los datos relativos a tipo de cambio esperado, tasas de interés doméstica y extranjeras, con el fin de estimar la curva de inflación esperada para el mercado local. El marco teórico está dado por las teorías de paridad (Emery, D., Finnerty, J., 2007), a saber:

a) Datos macroeconómicos. Estructura del tipo de interés en dos monedas: se proyecta la ETTI en moneda local y moneda extranjera, utilizando títulos en moneda doméstica y extranjera emitidos por el mismo país. La ETTI debe construirse con tasas contado las cuales no abundan para horizontes de mediano y largo plazo en mercados emergentes. Las técnicas comunes de implementación son el el bootstrapping ${ }^{8}$ y ajuste logarítmico. En este caso fue seleccionado el ajuste logarítmico ante la falta en el mercado de capitales local de tasas contado de mediano plazo, atento al proceso de renegociación de deuda.

\footnotetext{
${ }^{8} \mathrm{~A}$ partir de bonos cupón cero a un año, se puede derivar el resto de los plazos con STRIPS, en tanto y en cuanto estos tengan liquidez (mercado secundario). Si no se cumple el requisito de liquidez, el uso de STRIPS distorsiona la relación rendimientos y plazos de la curva spot. En esos casos, la técnica a la cual se recurre es el bootstrapping (Fabozzi, F., Fabozzi, D., 1996).
} 
b) Datos macroeconómicos. Proyección de tipos de cambio futuro e inflación domésti$c a$ : estimada la ETTI se aplican las teorías de paridad en los tipos de interés para proyectar la evolución del tipo de cambio, donde de interés local $\left(r_{t, d}\right)$ y extranjera $\left(r_{t, e}\right)$ explicando la relación entre el tipo de cambio contado (spot); (S) y el tipo de cambio futuro nominal (Ft).

$$
\frac{\mathrm{Ft}}{\mathrm{S}}=\frac{\left(1+r_{t, d}\right)}{\left(1+r_{t, e}\right)}(2)
$$

c) Datos macroeconómicos. Proyección de la tasa de inflación esperada doméstica: $\left(\pi_{t, d}\right)$. Se utiliza la teoría de la paridad en el poder de compra (PPC) partiendo de la ecuación 2 y la tasa de inflación esperada correspondiente al mercado desarrollado $\left(\pi_{t, e}\right)$.

$$
\frac{E\left[S_{t, d}\right]}{S}=\frac{\left(1+\pi_{t, d}\right)}{\left(1+\pi_{t, e}\right)}(3)
$$

Donde $E\left[S_{t}\right]$ representa el tipo de cambio esperado, y por la teoría de convergencia en futuros $E\left[S_{t}\right]=F_{t}$, (Hull, 2012). De la ecuación 3 se puede inferir la tasa de inflación proyectada doméstica por periodo.

$$
\pi_{t, d}=\frac{E[S]}{S} \times\left(1+\pi_{t, e}\right)-1(4)
$$

d) Datos macroeconómicos. Tasas nominales y reales proyectadas (efecto Fisher): ${ }^{\ominus}$ se establece la relación entre tasas de interés real y nominal.

$$
r_{t, d}=\left(r_{t, r}+\pi_{t, d}\right) \times\left[r_{t, r} \times \pi_{t, 4}\right](5)
$$

Las ecuaciones anteriores son el instrumento para resolver la falta de reserva de valor correspondiente a la unidad de medida doméstica, en especial, cuando son intercambiados flujos financieros temporales. Seguidamente se detallan las expresiones para obtener el valor de los flujos de fondos y la tasa de costo de capital.

\section{2- PROYECCIÓN DEL FLUJO DE FONDOS Y LOS FUNDAMENTOS DE VALOR}

A partir de los conductores de valor se proyecta el flujo de fondos en moneda de cierre, suponiendo un comportamiento estocástico de variables como la tasa de crecimiento del sector y la velocidad de convergencia del margen operativo. Los periodos de proyección son segrega- 
dos, iniciando un periodo "corona" 2020 con fuerte descenso de crecimiento y margen. Seguidamente comienza el periodo explícito de proyección el cual se divide en dos partes: la primera de recuperación cuya duración es función de la velocidad de ajuste del margen al nivel objetivo; la segunda parte consiste en mantener el nivel de margen operativo. Durante este periodo se supone que se mantienen las ventajas competitivas, posicionamiento en el sector y barreras de entrada y salida que justifican el margen objetivo. Finalmente se proyecta el valor de continuidad, las variables ingresan en un "steady state" donde se parte de la hipótesis de crecimiento en contexto de madurez de negocio y competencia perfecta, (Koller, T., Goedhart, M., Wessels, D., 2010); (Damodaran, A., 2012).

A partir de lo expuesto, la expresión de flujo de fondos libres en moneda doméstica es:

$$
F F L_{t+1}=\left\{\left[\left(I_{t} \times E(g)_{t}\right) \times E(m g)_{t}\right] \times(1-T)-\Delta I_{t}\right\} \times\left[\left(1+E\left(\pi_{t d}\right)\right](6)\right.
$$

Seguidamente son desarrollados cada uno de los componentes que integran la ecuación anterior:

a. Ganancias antes de intereses y después de impuestos $\left[E B I T_{t} \times(1-T)\right]$ surge de tomar la cifra de ingresos $I_{t}$ y multiplicarla por el crecimiento real esperado para cada periodo $E(g)_{t}$. Cabe destacar que este crecimiento esperado será negativo en este periodo, para luego retomar valores normales.

b. El crecimiento real $E(g)_{t}$ : es una variable estocástica, la cual es simulada mediante Monte Carlo, suponiendo distribución normal $E(g)_{t}=$ aleatorio $(x)$, medida, desvio.

c. Margen operativo $E(m g)_{t}$ : es el producto del descenso de ingresos y estabilidad de costos fijos este es negativo $m g_{t}=E B I T_{t} / I_{t}$, valor negativo a ser utilizado en el primer año, pero que asciende y converge al margen objetivo de mediano y largo plazo del sector $m g\left(^{*}\right)$.

d. El momento de convergencia $\left(t^{*}\right)$ : se establece en base a los fundamentos del negocio conforme se expone en la ilustración 3 , siendo estimado como, si $t>t^{*} ; m g\left(^{*}\right)$;

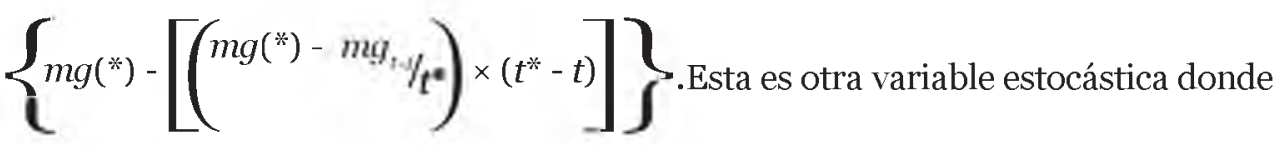

la velocidad de convergencia adopta un comportamiento uniforme entre dos valores donde $E(m g)_{t}=$ aleatorio $(x 1, x 2)$.

e. La tasa marginal de impuestos $T$ : se aplica en el caso de que exista ganancia imponible, caso contrario se deben tener en consideración los resultados negativos $R N_{t}$, pues los primeros periodos los tendrán por lo tanto $\operatorname{si}_{E B I T_{t}}>0 ;\left\{\left[E B I T_{t}<R N_{t-1} ; E B I T_{\mathrm{t}} ; E B I T_{t}\right.\right.$

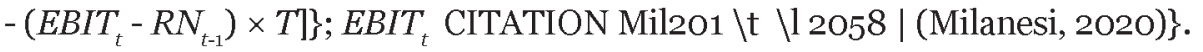


f. La inversión incremental $\Delta I_{t}$ : surge a partir de la tasa de rotación de la inversión total o activos del negocio A, que surge del ratio $r\left({ }^{*}\right)=V / A$ histórico, tal

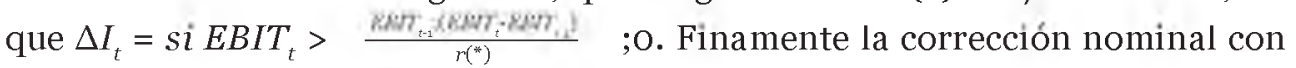
el objeto de expresar los flujos en moneda de cierre se realiza con la inflación proyectada para cada periodo, $E\left(\pi_{t_{d}}\right)$.

g. La inflación proyectada $E\left(\pi_{t d d}\right)$ : estimada a partir de la ecuación 4 .

\section{3- TASA DE COSTO DEL CAPITAL}

La tasa del costo del capital promedio ponderado se estima en dólares estadounidenses para luego convertirse en moneda doméstica, siguiendo el procedimiento expuesto en el anexo 1, gráfico A.1.1. En el caso de los mercados emergentes e incompletos existen situaciones en donde no se verifican precios por riesgos, tornando inconveniente la estimación de la tasa en moneda doméstica, es por ello que se trabaja en moneda dura siguiendo la lógica del "build-up model"(Pratt, S., Grabowski, R., 2008); (Damodaran, 2009); (Damodaran, A., 2015). Los cimientos se encuentran en el modelo CAPM (Capital Asset Pricing Model) adaptado a emergentes (Damodaran, A., 2009). En estos modelos la prima por riesgo de mercado global o de Estados Unidos, $E\left(r_{m}-r_{f}\right)$ se ajusta por el riesgo país correspondiente al mercado objetivo $\left(r_{p}\right)$. El coeficiente beta $(\beta)$ es calculado a partir de los sectoriales no apalancados y ajustándolos por la estructura deuda-capital correspondiente al objetivo de valuación. La ecuación correspondiente al costo de capital propio es la siguiente:

$$
E\left(k_{e(t)}\right)=r_{f(t)}+\left[r_{p(t)}+E\left(r_{m(t)}-r_{f(t)}\right)\right] \times \beta_{c}(7)
$$

La tasa de costo de la deuda en moneda dura, se estima a partir de la tasa de deuda en el mercado internacional, en función a calificación crediticia, según la relación de cobertura de intereses proyectada. Dicha tasa se ajusta por el correspondiente ahorro fiscal. La tasa esperada para cada periodo del costo promedio ponderado incorpora los costos mencionados y la estructura objetivo de capital (Damodaran, A., 2015). Estas a valores contables, pues estos marcan los fundamentos de la decisión de mezcla correspondiente a la firma.

$$
E\left(\operatorname{ccpp}_{t(u s)}\right)=W_{1} \times E\left(k_{e(t)}\right)+W_{1} \times E\left(k_{i(t)}\right)(8)
$$

El costo del capital en moneda extranjera se convierte a moneda dura mediante la siguiente expresión: 


$$
E\left(c c p p_{t(\$)}\right)=\left[1+E\left(c c p p_{t(\Phi)}\right)\right] \times \frac{\left(1+\pi_{t, d}\right)}{\left(1+\pi_{t . e^{2}}\right)}(9)
$$

La expresión anterior permite estimar un costo esperado del capital en moneda doméstica a partir de precios de riesgo generados en mercados desarrollados, enmendando en parte los problemas de información propios de economías emergentes.

\section{4- VALUACIÓN MEDIANTE EL DESCUENTO DE FLUJOS DE FONDOS}

Los flujos de fondos esperados en moneda dura se obtienen del cociente de las ecuaciones 6 y $2, \mathrm{E}\left(\mathrm{FF}_{\mathrm{t}(\mathrm{U})}\right)=\mathrm{E}\left(\mathrm{FF}_{\mathrm{t}}\right) / F_{t}$. Utilizando como factor de descuento el costo del capital estimado en la ecuación 8 se tiene el valor de la firma en marcha o valor intrínseco en dólares.

$$
V_{t=0}=\sum_{t=1}^{n} \frac{E\left(F F_{t(u \S)}\right)}{\left(1+c c p p_{t(u \uparrow)}\right)^{t}}=\frac{E\left(F F_{t+1(u \$)}\right)}{\left(1+c c p p_{t+1}(u \$)\right)^{t+1}}+\frac{E\left(F F_{t+2}(u \$)\right)}{\left(1+c c p p_{t+2}(u \$)\right)^{t / 2}}+\ldots+\frac{\frac{E\left(F F_{n}(u \$)\right)}{\left(c c p p_{n(u \$)}-g n\right)^{t+2}}}{\left(1+c c p p_{n}(u \$)\right)^{n}}(9)
$$

El valor obtenido se debe ajustar por el valor esperado de fracaso financiero $\mathrm{VQ}_{\mathrm{t}=0}$, que surge del producto entre las probabilidades de insolvencia $p_{(x)}$ y el valor de la firma $V L_{t=0}$ (Damodaran, 2009)..$^{10}$

$$
\mathrm{VQ}_{\mathrm{t}=\mathrm{o}}=E\left[p_{(x)}\right] \times V L_{t=0}(10)
$$

Conformesurge dela hoja de ruta contenida en el gráfico3, las probabilidades son obtenidas de una simulación Monte Carlo, suponiendo una distribución normal $p_{(x)}=$ aletorio $(x)$,media,desvio

Al valor intrínseco operativo se suman los valores de mercado correspondientes a el efectivo e inversiones transitorias Cash $+\mathrm{IT}_{\mathrm{t}=\mathrm{o}} \mathrm{y}$ los valores correspondientes a activos no operativos de largo plazo ANOLP $_{\mathrm{t}=0^{\circ}}$.

$$
\operatorname{Vadj}_{t=0}=\left[\left(V_{t=0}-V Q_{t=0}\right)+\left(C a s h+I T_{t=0}\right)+A N O L P_{t=0}\right](11)
$$

El valor correspondiente al capital contable se arriba deduciendoel valor actual dela deuda financiera $\left(D_{t=0}\right)^{11}$ y de las participaciones minoritarias (crowfunding) $P_{t=0}$, siendo $\operatorname{VPN}_{\mathrm{t}=\mathrm{o}}=\operatorname{Vadj}_{\mathrm{t}=\mathrm{o}}-D-\mathrm{P}$.

\footnotetext{
${ }^{10}$ La base puede ser el valor de libros o el valor intrínseco. Por lo general se toma el valor de libros para calcular el probable valor de quiebra.

${ }^{11}$ A menudo se computa la deuda comercial, esta es un concepto operativo que hace a la dimensión del activo de la firma. La deuda que define la estructura objetivo es la financiera aplicada desde el mercado de capitales (bancaria, mercado de dinero y títulos valores)
} 


\section{4- EL VALOR INTRÍNSECO DE LAS EMPRESAS COTIZANTES EN EL MERCADO ARGENTINO EN TIEMPOS DE COVID-19}

Seguidamente el modelo de valuación desarrollado en el apartado anterior será aplicado sobre el conjunto de firmas cotizantes en el mercado de capitales argentinos ${ }^{12}$, la información presentada es al 12 de junio del 2020. La tabla 2. presenta los valores correspondientes a las principales variables financieras de mercado a fecha de medición.

Tabla 2: Variables financieras macroeconómicas al 16/06/2020.

\begin{tabular}{|ccccc|ccc|c|}
\hline \multirow{2}{*}{ LIBOR 180 $\left.{ }^{13}\right)$} & LIBOR 90 $\left.{ }^{13}\right)$ & LIBOR 30 $\left({ }^{13}\right)$ & \multirow{2}{*}{ BADLAR $\left({ }^{14}\right)$} & TC Bolsa $\left({ }^{14}\right)$ & \multicolumn{3}{|c|}{ CER $\left({ }^{15}\right)$} & \multicolumn{2}{l|}{ Riesgo País J } \\
& & & & $6-19$ & $5-20$ & Anual & JPMorgan EMBI $+\left({ }^{16}\right)$ \\
\hline $\mathbf{0 , 4 2 \%}$ & $\mathbf{0 , 3 1 \%}$ & $\mathbf{0 , 1 8 \%}$ & $\mathbf{3 4 , 0 5 \%}$ & $\mathbf{8} \mathbf{1 0 6 , 7 2}$ & $\mathbf{1 4 , 6}$ & $\mathbf{2 1 , 4}$ & $\mathbf{4 6 , 7 2 \%}$ & $\mathbf{2 5 7 3}$ \\
\hline
\end{tabular}

Fuente: elaboración propia en base a: IAMC, BCRA, RAVA S.A, BYMA S.A).

La curva de regresión logarítmica correspondiente a la proyección correspondiente al EMAE 2020 se encuentra expuesta en el anexo 2, gráfico A.2.1, siendo y = -0,028ln(x) + 0,0748. El gráfico A.2.2 presenta la evolución de la tasa de inflación de EE.UU durante los últimos 10 años. La siguiente tabla expone para EE.UU y Argentina la inflación, tasa libre de riesgo EE.UU bonos del tesoro 10 años y el EMAE. Este último es la tasa de crecimiento $E(g)_{t}$ del flujo de fondos libres a ser simulada.

Tabla 3: Variables inflación y tasas libre de riesgo EE.UU y actividad Argentina.

\begin{tabular}{|ll|}
\hline \multicolumn{2}{|c|}{ Estados Unidos } \\
\hline IPC 2020-2010 $\left({ }^{17}\right)$ & $1,65 \%$ \\
Federal Funds 10 y 2020-2010 $\left.{ }^{18}\right)$ & $2,28 \%$ \\
\hline \multicolumn{2}{|c|}{ Argentina } \\
\hline EMAE proyectado 2020 & $-13,83 \%$ \\
EMAE media geométrica anual 2019-2004 & $1,96 \%$ \\
\hline
\end{tabular}

Fuente: elaboración propia.

En el anexo 2 la tabla A.2 presenta los diferentes bonos domésticos en moneda de curso legal y dólares estadounidenses utilizados para la proyección de las tasas esperadas en pesos y dólares expuestas en la tabla 4. La técnica de proyección es mediante la curva de rendimientos, ajustando una curva logarítmica entre tasas de rendimiento y duración modificada (DM), conforme se expo-

\footnotetext{
${ }^{12}$ Las fuentes de información se corresponden con el sitio de la Bolsa de Comercio de Buenos Aires (www.bolsar.com), Instituto Argentino de Mercado de Capitales (www.iamc.com.ar) y los informes disponibles en el sitio Invertir Online (www.invertironline.com.ar), Banco Central de la República Argentina (www.bcra.gov.ar) y RAVA sociedad de bolsa S.A (www.rava.com)

${ }^{13}$ Fuente 16/06/2020 https://www.bcra.gob.ar/PublicacionesEstadisticas/libor.asp

${ }^{14}$ Fuente16/06/2020 https://iamcmediamanager.prod.ingecloud.com/mediafiles/iamc/2020/6_16/0/11/65/737739.pdf

${ }^{15}$ Fuente16/06/2020 http://www.bcra.gov.ar/PublicacionesEstadisticas/Principales_variables_datos.asp

${ }^{16}$ Fuente16/06/2020 https://www.rava.com/empresas/perfil.php?e=RIESGO\%20PAIS\&gclid=EAlaIQobChMlyvHu9qjq5wIVI4WR ChO VA7rEAAYASAAEgKakPD_BwE

17 Fuente 16/06/2020 https://es.global-rates.com/estadisticas-economicas/inflacion/indice-de-precios-al-consumo/ipc/estadosunidos.aspx

${ }^{18}$ Fuente 16/06/2020 https://www. macrotrends, net/2016/10-year-treasury-bond-rate-yield-chart

${ }^{19}$ Fuente 16/06/2020 https://www.indec.gob.ar/indec/web/Nivel4-Tema-3-9-48
} 
ne en los gráficos A.2.3 y A.2.4 respectivamente. Cabe destacar que el tipo de cambio futuro se obtiene aplicando las ecuaciones 2 y 3. La inflación proyectada para Argentina (ante última columna) surge de aplicar la ecuación 4, conforme se expone a continuación:

Tabla 4: Variables inflación y tasas libre de riesgo EE.UU y actividad Argentina.

\begin{tabular}{|c|c|c|c|c|c|c|c|c|}
\hline \multirow{2}{*}{$\begin{array}{c}\mathrm{t} \\
31 / 12 / 2019\end{array}$} & \multirow{2}{*}{$\begin{array}{l}\mathrm{E}(\mathrm{i}) \mathrm{FF} \text { 10y } \\
\text { promedio }\end{array}$} & \multirow{2}{*}{$\begin{array}{c}\mathrm{E}(\mathrm{i}) \\
\text { bonos } \$\end{array}$} & \multirow{2}{*}{$\begin{array}{c}\mathrm{E}(\mathrm{i}) \\
\text { bonos u\$ }\end{array}$} & \multicolumn{2}{|c|}{$\mathrm{E}(\mathrm{F})$} & \multirow{2}{*}{$\begin{array}{l}\mathrm{E}(\pi) \mathrm{EEU} \\
\text { promedio }\end{array}$} & \multirow{2}{*}{$\begin{array}{c}\mathrm{E}(\pi) \\
\text { Argentina }\end{array}$} & \multirow[t]{2}{*}{$\mathrm{E}$ (i real) } \\
\hline & & & & $\$$ & 80,00 & & & \\
\hline $31 / 12 / 2020$ & $2,28 \%$ & $81,00 \%$ & $64,13 \%$ & $\$$ & 141,57 & $1,65 \%$ & $79,87 \%$ & $0,62721 \%$ \\
\hline $31 / 12 / 2021$ & $2,28 \%$ & $60,83 \%$ & $47,29 \%$ & $\$$ & 222,60 & $1,65 \%$ & $59,83 \%$ & $0,62721 \%$ \\
\hline $31 / 12 / 2022$ & $2,28 \%$ & $49,03 \%$ & $37,43 \%$ & $\$$ & 324,34 & $1,65 \%$ & $48,10 \%$ & $0,62721 \%$ \\
\hline $31 / 12 / 2023$ & $2,28 \%$ & $40,66 \%$ & $30,44 \%$ & $\$$ & 446,03 & $1,65 \%$ & $39,78 \%$ & $0,62721 \%$ \\
\hline $31 / 12 / 2024$ & $2,28 \%$ & $34,17 \%$ & $25,02 \%$ & $\$$ & 585,07 & $1,65 \%$ & $33,33 \%$ & $0,62721 \%$ \\
\hline $31 / 12 / 2025$ & $2,28 \%$ & $28,86 \%$ & $20,59 \%$ & $\$$ & 737,09 & $1,65 \%$ & $28,06 \%$ & $0,62721 \%$ \\
\hline $31 / 12 / 2026$ & $2,28 \%$ & $24,37 \%$ & $16,84 \%$ & $\$$ & 896,29 & $1,65 \%$ & $23,60 \%$ & $0,62721 \%$ \\
\hline $31 / 12 / 2027$ & $2,28 \%$ & $20,49 \%$ & $13,60 \%$ & $\$$ & $1.055,82$ & $1,65 \%$ & $19,74 \%$ & $0,62721 \%$ \\
\hline $31 / 12 / 2028$ & $2,28 \%$ & $17,06 \%$ & $10,74 \%$ & $\$$ & $1.208,37$ & $1,65 \%$ & $16,33 \%$ & $0,62721 \%$ \\
\hline $31 / 12 / 2029$ & $2,28 \%$ & $13,99 \%$ & $8,18 \%$ & $\$$ & $1.346,73$ & $1,65 \%$ & $13,28 \%$ & $0,62721 \%$ \\
\hline $31 / 12 / 2030$ & $2,28 \%$ & $11,22 \%$ & $5,86 \%$ & $\$$ & $1.464,42$ & $1,65 \%$ & $10,53 \%$ & $0,62721 \%$ \\
\hline
\end{tabular}

Fuente: elaboración propia.

La tabla 4. brinda los insumos para proyectar la tasa del costo del capital dólares y pesos conforme surge del esquema contenido en el Anexo 1, gráfico A.1.1 y las ecuaciones 7, 8 y 9. El resultado se expone en las tablas 5,6 y 7 .

Tabla 5: Tasa libre de riesgo, adicional por riesgo de mercado, calificación crediticia.

\begin{tabular}{|c|c|c|c|c|c|c|c|c|c|}
\hline \multirow{2}{*}{ Periodos } & \multirow[t]{2}{*}{$\mathrm{E}(\mathrm{rf})$} & \multirow{2}{*}{$\begin{array}{l}\text { Argentina default } \\
\text { Spread Ajustado }^{20}\end{array}$} & \multirow{2}{*}{$\begin{array}{c}\text { S\&P } 500 \\
\text { MRP }\end{array}$} & \multirow{2}{*}{\multicolumn{2}{|c|}{ Ratio Ganancia/Intereses }} & \multicolumn{2}{|c|}{ Calificacion Moodys } & \multicolumn{2}{|c|}{ Adicional por riesgo en u\$ } \\
\hline & & & & & & Empresa & Argentina & Empresa & Argentina \\
\hline $31 / 12 / 2020$ & $2,28 \%$ & $18,45 \%$ & $6,01 \%$ & -100000 & 0,199999 & $\mathrm{D} 2 / \mathrm{D}$ & $\mathrm{Ca}$ & $28,34 \%$ & $15,00 \%$ \\
\hline $31 / 12 / 2021$ & $2,28 \%$ & $11,86 \%$ & $6,01 \%$ & -100000 & 0,199999 & $\mathrm{D}_{2} / \mathrm{D}$ & $\mathrm{B}_{3}$ & $28,34 \%$ & $9,65 \%$ \\
\hline $31 / 12 / 2022$ & $2,28 \%$ & $11,86 \%$ & $6,01 \%$ & 0,65 & 0,799999 & $\mathrm{Ca} 2 / \mathrm{CC}$ & $\mathrm{B}_{3}$ & $16,20 \%$ & $9,65 \%$ \\
\hline $31 / 12 / 2023$ & $2,28 \%$ & $11,86 \%$ & $6,01 \%$ & 0,8 & 1,249999 & $\mathrm{C} 2 / \mathrm{C}$ & $\mathrm{B} 2$ & $15,37 \%$ & $8,16 \%$ \\
\hline $31 / 12 / 2024$ & $2,28 \%$ & $11,86 \%$ & $6,01 \%$ & 1,25 & 1,499999 & $\mathrm{~B}_{3} / \mathrm{B}-$ & $\mathrm{B}_{2}$ & $9,65 \%$ & $8,16 \%$ \\
\hline $31 / 12 / 2025$ & $2,28 \%$ & $11,86 \%$ & $6,01 \%$ & 1,25 & 1,499999 & $\mathrm{~B}_{3} / \mathrm{B}-$ & $\mathrm{B} 2$ & $9,65 \%$ & $8,16 \%$ \\
\hline $31 / 12 / 2026$ & $2,28 \%$ & $10,04 \%$ & $6,01 \%$ & 1,25 & 1,499999 & $\mathrm{~B}_{3} / \mathrm{B}-$ & $\mathrm{B}_{1}$ & $9,65 \%$ & $6,68 \%$ \\
\hline $31 / 12 / 2027$ & $2,28 \%$ & $10,04 \%$ & $6,01 \%$ & 1,25 & 1,499999 & $\mathrm{~B}_{3} / \mathrm{B}-$ & $\mathrm{B}_{1}$ & $9,65 \%$ & $6,68 \%$ \\
\hline $31 / 12 / 2028$ & $2,28 \%$ & $8,21 \%$ & $6,01 \%$ & 1,25 & 1,499999 & $\mathrm{~B}_{3} / \mathrm{B}-$ & $\mathrm{B}_{1}$ & $9,65 \%$ & $6,68 \%$ \\
\hline $31 / 12 / 2029$ & $2,28 \%$ & $8,21 \%$ & $6,01 \%$ & 2,5 & 2,999999 & Baaz/BBB & $\mathrm{B}_{1}$ & $4,50 \%$ & $6,68 \%$ \\
\hline $31 / 12 / 2030$ & $2,28 \%$ & $8,21 \%$ & $6,01 \%$ & 2,5 & 2,999999 & $\mathrm{Baa2} / \mathrm{BBB}$ & $\mathrm{Ba} 2$ & $4,50 \%$ & $4,45 \%$ \\
\hline
\end{tabular}

Fuente: elaboración propia en base las tablas de A. Damodaran.

\footnotetext{
${ }^{20}$ Fuente 16/06/2020 http://pages.stern.nyu.edu/ adamodar/New_Home_Page/datafile/ctryprem.html
} 
En la tabla 5. se utiliza como tasa libre de riesgo el rendimiento promedio aritmético (20192009) de los bonos del tesoro de EE.UU. a 10 años $r_{f}$, el adicional por riesgo de mercado para EE.UU según el índice S\&P 500 promedio, el adicional por riesgo país Argentina ajustado por volatilidad (cociente entre volatilidad renta variable-renta fija). Además, las calificaciones Moodys sector privado y soberando, suponiendo un escenario donde existe una renegociación satisfactoria de la deuda pública con tenedores de títulos. Estas sirven de base para proyectar la tasa de costo de capital ajeno, suponiendo que la firma a medida que recupera los niveles de margen operativo y EBIT, mejora el margen de cobertura de intereses y su calificación crediticia en dólares de EE.UU.

Tabla 6: Drivers de valor e información de mercado empresas locales marzo 2020.

\begin{tabular}{|c|rrrrrrr|}
\hline \multirow{2}{*}{ Sectores (21) } & Margen & Ratio & Vtas prom & Ratio & Vtas (i) & $\beta(1)$ & $\beta(\mathrm{u})$ \\
& Operativo & V/A & Prom $5 y y$ & D/PN & Vtas totales & \\
\hline Consumo básico & $5,83 \%$ & 1,59 & 15,5 & $57,80 \%$ & $2,13 \%$ & 0,47 & 0,33 \\
Consumo discrecional & $12,53 \%$ & 1,32 & 5,7 & $40,90 \%$ & $0,78 \%$ & 0,52 & 0,40 \\
Energía & $\mathbf{1 3 , 5 7 \%}$ & 5,31 & 329,1 & $54,36 \%$ & $45,16 \%$ & 0,64 & 0,46 \\
Industrial & $6,00 \%$ & 0,98 & $\mathbf{1 , 8}$ & $21,98 \%$ & $0,25 \%$ & 0,40 & 0,35 \\
Inmobiliario & $\mathbf{1 5 , 5 3 \%}$ & 0,14 & 21,8 & $0,00 \%$ & $2,99 \%$ & 0,55 & 0,55 \\
Materiales & $7,49 \%$ & 0,84 & 13,5 & $79,26 \%$ & $1,85 \%$ & 0,53 & 0,34 \\
Salud & $\mathbf{1 0 , 0 9 \%}$ & 0,9 & $\mathbf{1}$ & $66,32 \%$ & $0,14 \%$ & 0,25 & 0,17 \\
Servicios Públicos & $20,38 \%$ & 0,69 & 18,1 & $40,38 \%$ & $2,48 \%$ & 0,75 & 0,58 \\
Tecnología información & $13,34 \%$ & 0,46 & 1,6 & $5,46 \%$ & $0,22 \%$ & 0,77 & 0,74 \\
Telecomunicaciones & $7,31 \%$ & 0,44 & 320,6 & $61,66 \%$ & $44,00 \%$ & 0,48 & 0,34 \\
\hline Totales & $\mathbf{1 0 , 7 3 \%}$ & $\mathbf{2 , 6 8}$ & $7 \mathbf{2 8 , 7}$ & $\mathbf{5 5 , 8 6 \%}$ & $\mathbf{1 0 0 , 0 0 \%}$ & $\mathbf{0 , 5 1}$ & $\mathbf{0 , 3 7}$ \\
\hline
\end{tabular}

Fuente: elaboración propia en base las tablas BCBA, IMAC, Invertironline.

La tabla 6. expone los drivers de valor de cada sector a marzo 2020, sin perjuicio que la valoración se realiza a junio 2020, suponiendo un periodo irregular de medio año ${ }^{22}$. En el caso de la tasa de costo de capital las últimas columnas arrojan el coeficiente beta apalancado y sin deuda, obtenido del promedio ponderado correspondientes a betas sectoriales. Para la ponderación simplemente se tomaron las ventas promedio, en miles de millones de pesos, correspondientes a los últimos 5 años.

La tabla 7. aplica la ruta del costo del capital y las tasas de inflación proyectadas con el objeto de obtener costos promedios ponderados del capital en moneda dura y local.

La proyección de los flujos de fondos y sus variables se realiza con los datos de partida contenidos en la tabla 8 . El horizonte de proyección se segrega en cuatro partes: periodo 2020, un horizonte explícito con velocidad de convergencia a margen operativo de 3 años (2021-2024), un horizonte explícito de crecimiento (2025-2029) y valor de continuidad

\footnotetext{
${ }^{21}$ Fuente 16/06/2020 https://www. invertironline.com/research/reportes/1/informe-de-ratios/23/

${ }^{22}$ En tal sentido las magnitudes financieras se tomaron por medio año (desde julio a diciembre) y las tasas se proporcionaron a su equivalente semestral
} 
(2030 en adelante). El flujo de fondos (ecuación 6) emplea los drivers y fundamentos de valor (tabla 6), la tasa de crecimiento proyectada para el 2020 y años siguientes (tabla 3) y la tasa de inflación esperada como el tipo de cambio esperado (tabla 4), para su conversión a moneda dura.

Tabla 7: Drivers de valor e información de mercado empresas locales marzo 2020.

\begin{tabular}{|c|c|c|c|c|c|c|c|c|c|c|c|c|}
\hline \multirow{2}{*}{$t$} & \multirow{2}{*}{$\mathrm{B}(\mathrm{l})$} & \multirow{2}{*}{ B sec $(u)(23)$} & \multicolumn{2}{|c|}{ Estructura objetivo( $\left.{ }^{21}\right)$} & \multirow{2}{*}{$\begin{array}{c}\text { Tasa } \\
\text { Impuesto }\end{array}$} & \multirow{2}{*}{$\begin{array}{l}\mathrm{ke} \\
\mathrm{u} \$\end{array}$} & \multirow{2}{*}{$\begin{array}{l}\text { ke } \\
\$\end{array}$} & \multirow{2}{*}{$\begin{array}{c}\text { ke } \\
\$ \text { real }\end{array}$} & \multirow{2}{*}{$\begin{array}{l}\mathrm{ki} \\
\mathrm{u} \$\end{array}$} & \multirow{2}{*}{$\begin{array}{l}\text { CCPP } \\
\text { en u\$ }\end{array}$} & \multirow{2}{*}{$\begin{array}{l}\text { CCPP } \\
\text { en } \$\end{array}$} & \multirow{2}{*}{$\begin{array}{c}\text { CCPP } \\
\text { en \$ real }\end{array}$} \\
\hline & & & Wd & We & & & & & & & & \\
\hline 1 & 0,51 & 0,40 & 0,279 & 0,721 & $30 \%$ & $20,37 \%$ & $113,01 \%$ & $18,42 \%$ & $43,34 \%$ & $23,15 \%$ & $117,93 \%$ & $21,16 \%$ \\
\hline 2 & 0,51 & 0,40 & 0,279 & 0,721 & $30 \%$ & $15,02 \%$ & $80,85 \%$ & $13,15 \%$ & $37,99 \%$ & $18,25 \%$ & $85,93 \%$ & $16,33 \%$ \\
\hline 3 & 0.51 & 0,40 & 0,279 & 0,721 & $30 \%$ & $15,02 \%$ & $67,58 \%$ & $13,15 \%$ & $25,85 \%$ & $15,88 \%$ & $68,83 \%$ & $14,00 \%$ \\
\hline 4 & 0,51 & 0,40 & 0,279 & 0,721 & $30 \%$ & $13,53 \%$ & $56,13 \%$ & $11,69 \%$ & $23,53 \%$ & $14,35 \%$ & $57,26 \%$ & $12,50 \%$ \\
\hline 5 & 0,51 & 0,40 & 0,279 & 0,721 & $30 \%$ & $13,53 \%$ & $48,92 \%$ & $11,69 \%$ & $17,81 \%$ & $13,23 \%$ & $48,53 \%$ & $11,40 \%$ \\
\hline 6 & 0,51 & 0,40 & 0,279 & 0,721 & $30 \%$ & $13,53 \%$ & $43,03 \%$ & $11,69 \%$ & $17,81 \%$ & $13,23 \%$ & $42,66 \%$ & $11,40 \%$ \\
\hline 7 & 0,51 & 0,40 & 0,279 & 0,721 & $30 \%$ & $12,05 \%$ & $36,25 \%$ & $10,23 \%$ & $16,33 \%$ & $11,87 \%$ & $36,04 \%$ & $10,06 \%$ \\
\hline 8 & 0,51 & 0,40 & 0,279 & 0,721 & $30 \%$ & $12,05 \%$ & $31,99 \%$ & $10,23 \%$ & $16,33 \%$ & $11,87 \%$ & $31,79 \%$ & $10,06 \%$ \\
\hline 9 & 0,51 & 0,40 & 0,279 & 0,721 & $30 \%$ & $12,05 \%$ & $28,23 \%$ & $10,23 \%$ & $16,33 \%$ & $11,87 \%$ & $28,04 \%$ & $10,06 \%$ \\
\hline 10 & 0,51 & 0,40 & 0,279 & 0,721 & $30 \%$ & $12,05 \%$ & $24,88 \%$ & $10,23 \%$ & $11,18 \%$ & $10,87 \%$ & $23,56 \%$ & $9,07 \%$ \\
\hline 11 & 0,5138 & 0,40 & 0,279 & 0,721 & $30 \%$ & $9,83 \%$ & $19,42 \%$ & $8,05 \%$ & $8,95 \%$ & $8,83 \%$ & $18,34 \%$ & $7,07 \%$ \\
\hline
\end{tabular}

Fuente: elaboración propia en base las tablas BCBA, IMAC, Invertironline.

Tabla 8: Supuestos de proyección iniciales.

\begin{tabular}{|c|c|c|}
\hline Fundamentos de valor & \multicolumn{2}{|c|}{ Variables } \\
\hline Ventas último año totales en miles de millones de pesos $\left({ }^{20}\right)$ & $\$$ & $3.407,96$ \\
\hline Margen operativo Base $\left({ }^{20}\right)$ & & $10,73 \%$ \\
\hline Velocidad de convergencia (variable a simular) & & 3 \\
\hline Margen operativo de convergencia $\left({ }^{20}\right)$ & & $10,73 \%$ \\
\hline Activo valor de libros $\left({ }^{20}\right)$ & $\$$ & $9.124,54$ \\
\hline Patrimonio neto valor libros $\left({ }^{20}\right)$ & $\$$ & $6.576,21$ \\
\hline Deuda Valor libros $\left({ }^{20}\right)$ & $\$$ & $2.548,32$ \\
\hline Efectivo y Titulos valores negociables (supuesto $5 \%$ sobre B) & $\$$ & 456,23 \\
\hline Cross holdings (inversiones permanentes) y otros activos no operativos ( $15 \%$ sobre B) & $\$$ & $1.368,68$ \\
\hline Probabilidad de insolvencia (variable a simular) & & $10,00 \%$ \\
\hline Valor de ajuste insolvencia $\mathrm{Libro}=\mathrm{B}$, Intrínseco $=\mathrm{V}$ & & $\mathbf{V}$ \\
\hline
\end{tabular}

Fuente: elaboración propia.

Las proyecciones puntuales de aplicar el modelo integral de valuación (ecuaciones 9, 10 y 11) arrojan en miles de millones los siguientes valores para el mercado local expuestos en la tabla 9. 
Tabla 9: Valores Activos y Equity empresas locales al 12/06/2020.

\begin{tabular}{|l|c|l|c|}
\hline \multicolumn{1}{|c|}{ Valuación 12/06/2020 } & $\begin{array}{c}\text { Dólar } \\
\text { EE.UU }\end{array}$ & \multicolumn{1}{|c|}{ Valuación 12/o6/2020 } & $\begin{array}{c}\text { Moneda } \\
\text { Local }\end{array}$ \\
\hline PV Valor terminal & 6,41 & PV Valor terminal & 682,4 \\
PV Flujo de fondos & 8,96 & PV Flujo de fondos & 953,9 \\
Valor Activos Operativos & $\mathbf{1 5 , 3 8}$ & Valor Activos Operativos & $\mathbf{1 6 3 6 , 3}$ \\
(+) Efectivo y Titulos valores negociables u\$ & 4,29 & (+) Efectivo y Titulos valores negociables u\$ & 456,2 \\
(+) Cross holdings (inversiones permanentes) & $\mathbf{1 2 , 8 6}$ & (+) Cross holdings (inversiones permanentes) & 1368,7 \\
y otros activos no operativos u\$ & & y otros activos no operativos \$ & $\mathbf{3 4 6 1 , 2}$ \\
\hline Valor total de los activos & $\mathbf{3 2 , 5 2}$ & Valor total de los activos & $\mathbf{1 6 3 , 6}$ \\
\hline Ajuste insolvencia & $\mathbf{1 , 5 4}$ & Ajuste insolvencia & 2548,3 \\
(-) Deuda & $\mathbf{2 3 , 9 5}$ & (-) Deuda & $\mathbf{7 4 9 , 3}$ \\
\hline Valor Equity & $7, \mathbf{0 4}$ & Valor Equity & 749,3 \\
\hline Control Equity \$/Equity u\$ (dólar bolsa) & 106,42 & Control Equity u\$*TC Bolsa 12/o6/2020 & \multicolumn{2}{|c|}{} \\
\hline
\end{tabular}

Fuente: elaboración propia.

El valor total de los activos asciende a u\$ 32.52 (miles de millones) o $\$ 3461,2$ (miles de millones de pesos) con un dólar bolsa de paridad de u\$106,42 al 12 de junio del 2020.

Tabla 10: Valores Activos y Equity empresas locales pre COVID-19.

\begin{tabular}{|lrr|}
\hline \multicolumn{2}{|c|}{ Firma pre COVID-19 suponiendo steady state al 12/2019 } \\
\hline Flujo de fondos libres en u\$ & $\$$ & 3,20 \\
Tasa B2 Moody ratings & & $13,04 \%$ \\
Crecimiento & $\mathbf{1 , 9 6 \%}$ \\
Valor Intrínseco (VA) & $\$$ & 29,44 \\
(+) Efectivo y Titulos valores negociables u\$ & $\$$ & 5,70 \\
(+) Cross holdings (inversiones permanentes) y otros activos no operativos u\$ & $\$$ & 17,11 \\
\hline Valor total de los activos pre COVID-19 & $\$$ & $\mathbf{5 2 , 2 5}$ \\
\hline Perdida en u\$ & & $37,76 \%$ \\
\hline
\end{tabular}

Fuente: elaboración propia.

Si la pandemia y otros acontecimientos no se hubiesen desencadenado y asumiendo que las firmas se comportaban estables a perpetuidad, el valor de los activos sería de u\$52.25 (miles de millones) evidenciando una pérdida en dólares de valor del 38\% para las firmas locales. El mapa del valor expuesto en el gráfico 3 se reproduce en números en el anexo A.1.2.

La tabla 10. arroja el valor obtenido mediante proyecciones puntuales que conduce a obtener el valor ajustado por riesgo. La anatomía y estructura del riesgo surge de la simulación Montecarlo, para la cual fueron seleccionadas las siguientes variables aleatorias: 
a) Crecimiento real $E(g)_{\mathrm{t}}=$ aletorio $(x)$,media,desvio, suponiendo una distribución normal, con media $-13.85 \%$ y desvío $5 \%$ para el periodo 2020; media $1.96 \%$ y desvío 0.5\% para los periodos 2021 a 2025, media $1.96 \%$ y desvío $0.1 \%$ para los periodos 2026 a 2030.

b) Velocidad de convergencia $=$ si $t>t^{*} ; \operatorname{mg}(*) ;\left\{m g\left({ }^{*}\right)-\left[\left(m g_{t-1} / t^{*}\right) \times\left(t^{*}-\mathrm{t}\right)\right]\right\}$, de distribución uniforme con extremos 2 y 4 años.

c) Probabilidad de insolvencia $=E\left[p_{(x)}\right]$ suponiendo una distribución normal, con media $-10 \%$ y desvío $10 \%$.

La tabla 10. arroja el valor obtenido mediante proyecciones puntuales que conduce a obtener el valor intrínseco o teórico. La tabla 11. expone las salidas y resultados de la simulación realizada con MS Excel $®$ con la función números aleatorios. Los números se corresponden con el valor de los activos operativos en términos de estructura del riesgo (media, mediana, rango, desvío y coeficiente de variación). La probabilidad de que el valor sea mayor a u\$14 (miles millones) es del $84,15 \%$, con una probabilidad significativa del $16 \%$ de un valor inferior.

Tabla 11: Salidas de simulación 2000 iteraciones MS Excel @.

\begin{tabular}{|c|c|c|}
\hline Media & $u \$$ & 3,20 \\
\hline Mediana & u\$ & $13,04 \%$ \\
\hline Máximo & $\mathrm{u} \$$ & $1,96 \%$ \\
\hline Mínimo & $u \$$ & 29,44 \\
\hline Asimetria & \multicolumn{2}{|c|}{0,055626873} \\
\hline Curtosis & \multicolumn{2}{|c|}{$-0,529489672$} \\
\hline Desvío & $u \$$ & 1,48 \\
\hline $\mathrm{CV}$ & & 0,096168512 \\
\hline Probabilidad $V \geq u \$ 14$ & & $84,15 \%$ \\
\hline
\end{tabular}

Fuente: elaboración propia.

\section{5- CONCLUSIONES}

La actual situación pandémica puede calificarse como un típico cisne negro, imprevisto y con efectos dispares en los mercados y segmentos de negocios. Justamente esto hace que, a diferencias de otras crisis, el COVID-19 tiene ganadores y perdedores. Entre los segundos se encuentran aquellos negocios con mercados objetivos centrados en el transporte, ocio, esparcimiento o consumos discrecionales y cuya ecuación de valor es función del uso intensivo de capital fijo, 
factor humano para operaciones, con altas necesidades de efectivo y significativo apalancamiento. A nivel de valuación de empresas más que nunca se debe prestar atención a los fundamentos de valor, atentos a los nuevos formatos de negocios que el contexto actual impone.

En tal sentido se propone un modelo cuya filosofía reside en incorporar los fundamentos de valor en los flujos y los riesgos sistémicos en las tasas. Los fundamentos de valor miran la extrema situación del ejercicio presente proyectando recuperaciones graduales a niveles objetivos, ajustada a la velocidad de reacción del sector. Crecimiento, velocidad de recuperación del margen y probabilidad de insolvencia constituyen variables aleatorias proyectadas mediante simulación Monte Carlo. En particular el crecimiento y la velocidad de recuperación del margen objetivo. Para economías emergentes no se debe perder de vista el impacto de las tasas esperadas de inflación sobre las magnitudes financieras proyectadas. Es menester trabajar en un marco de paridades de tipos de intereses y tipos de cambios.

El modelo aplicado sobre el mercado local evidencia una convergencia a tasas del costo del capital e inflación moderadas, en el mediano a largo plazo y una significativa destrucción de valor empresarial por la pandemia. Amén de este cisne negro, a futuro se debe recrear la confianza en el ambiente de negocios local, garantizando la estabilidad de normas y el respecto por la función reserva de valor de la moneda local.

\section{REFERENCIAS BIBLIOGRÁFICAS}

Argandoña, A. (2013). Irvin Fisher: un gran economista. (I. B. Navarra, Ed.) Working Paper WP-1082 http://www.iese.edu/research/pdfs/WP-1082.pdf, 1-44.

Booth, L. (2007). Capital Cash Flow, APV and Valuation. European Financial Management, 13(1), 29-48.

Copeland, T., Koller, K., Murrin, J. (2000). Valuation: Measuring and Managing the Value of Companies (3 ed.). New York: Wiley.

Damodaran. (2009). The Dark Side of Vahuation: Valuing Young, Distressed and Complex Business (Second ed.). USA: Prentice Hall.

Damodaran, A. (2006). Damodaran on Valuation (Second ed.). New York, United State: John Wiley \& Sons. 
Damodaran, A. (2009). Volatility Rules: Valuing Emerging Market Companies. Working Paper, http://people.stern.nyu.edu/adamodar/pdfiles/papers/emergmkts.pdf, 1-38.

Damodaran, A. (2012). Investment Valuation: tools and techniques for determining the value of any asset (3er ed.). NY: Wiley Finance Series.

Damodaran, A. (2015). Country Risk: Determinants, Measures and Implications The 2015 edition. (SSRN, Ed.) Social Science Research Network (SSRN). Downloads/SSRN-id2630871. $p d f, 1-97$.

Emery, D., Finnerty, J., (2007). Corporate Financial Management (3 ed.). New Jersey; NJ: Prentice Hall.

Fabozzi, F., Fabozzi, D., (1996). Bond Markets, Analysis and Strategies. New Jersey, NJ: Prentice Hall. Englewood Cliffs.

Fernández, P. (27 de junio de 2012). Valoración de empresas por descuento de flujos de fondos: lo fundamental y las complicaciones innecesarias. Obtenido de Social Science Research Network (SSRN): http://ssrn.com/abstrac=2089397

Fernández, P. (2014). Valoración de Empresas y Sensatez (Tercera ed.). Barcelona: IESE Business School-Universidad de Navarra.

Hull, J. (2012). Options, Futures and other Derivatives (Global Edition). Pearson Education Limited 8va edición.

Koller, T., Goedhart, M., Wessels, D. (2010). Valuation: measuring and managing the value of companies (5ta ed.). New Jersey: John Wiley \& Sons.

Milanesi, G. (2013). Teoría de Opciones: Modelos específicos y aplicaciones para valorar estrategias, activos reales e instrumentos financieros (Primera ed.). Bahía Blanca: Editorial de la Universidad Nacional del Sur. REUN.

Milanesi, G. (2017). Valuación de empresas: enfoque integral para mercados emergentes e inflacionarios. Estudios Gerenciales, 33(145), 377-390. 
Milanesi, G. (2018). Opciones reales y funciones isoelásticas: el caso de la valuación de un proyecto de I\&D en mercados incompletos. Revista Española de Capital de Riesgo(2), 39-52.

Milanesi, G. (2020). Opciones reales y el valor de los ahorros fiscales. Revista Ciencias Administrativas(16), 61-70.

Milanesi, G., Weins, G., Pequeño, D. (2020). Teoria de la paridad y valuación en dos monedas con descuento de flujos de fondos y lógica fuzzy. Estocástica: Finanzas y Riesgo, 10(1), 27-75.

Pratt, S., Grabowski, R. (2008). Cost Of Capital: Applications and Examples (3 ed.). New Jersey: John Wiley \& Sons.

Ruback, R. (2002). Capital Cash Flows: A simple approach to valuing risky cash flows. Financial Management, 31(2), 85-103.

\section{CURRICULUM VITAE}

\section{Gastón S. Milanesi}

Doctor en Ciencias de la Administración (UNS). Magister en Administración (UNS). Contador Público (UNS), Argentina.

milanesi@uns.edu.ar 


\section{ANEXO 1}

Gráfico A.1.1: La ruta del costo del capital en emergentes

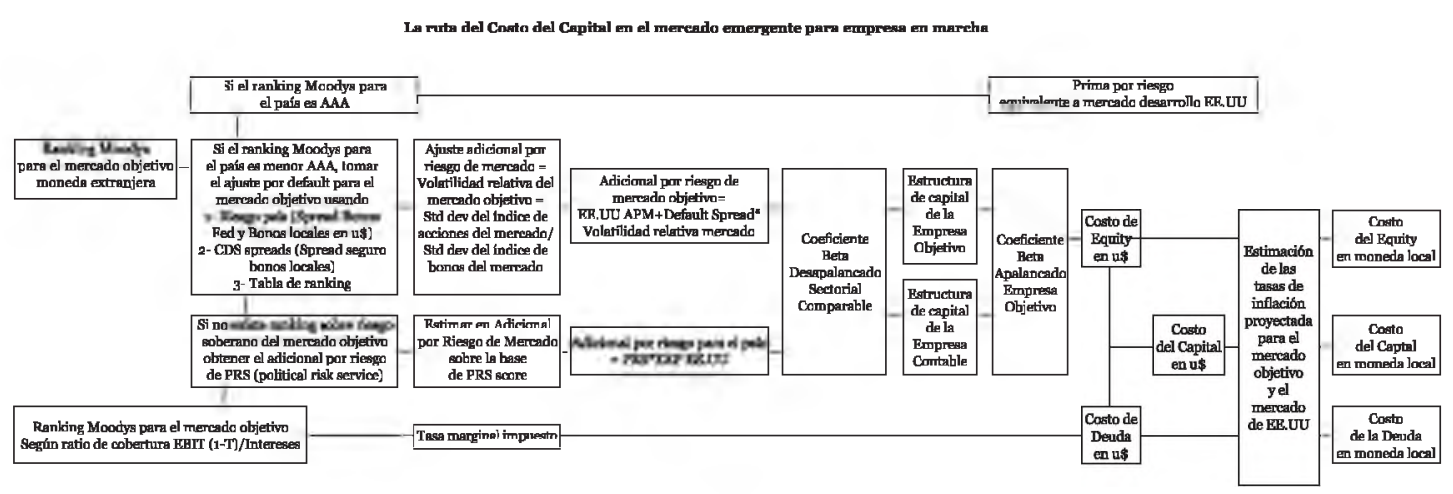

Fuente: elaboración propia.

Gráfico A.1.2: Contexto macro, drivers y valor en números

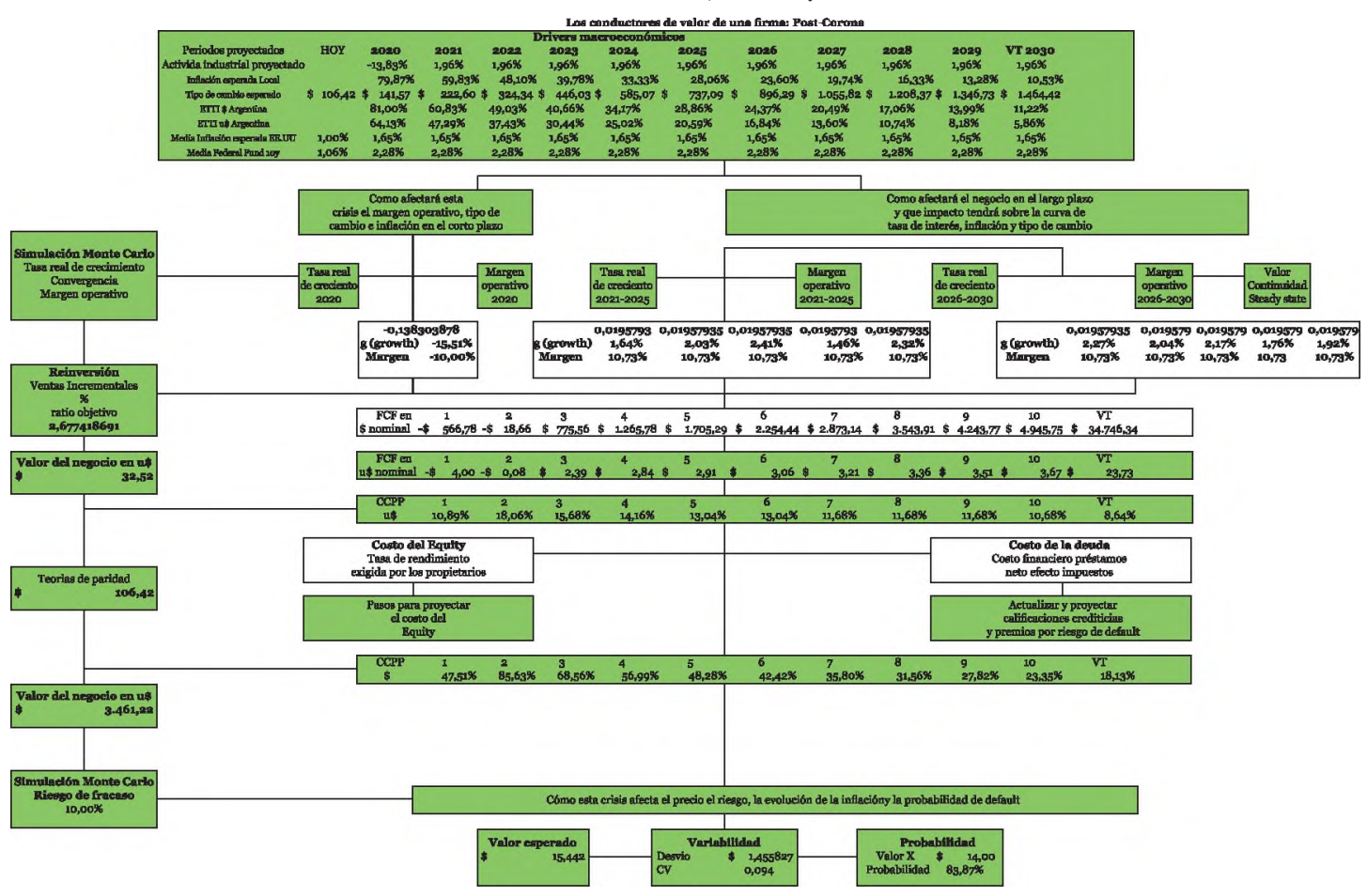

Fuente: elaboración propia. 


\section{ANEXO 2: Datos macroeconómicos}

Tabla A.2: Bonos soberanos al 12/06/2020

\begin{tabular}{|llcc|llc|}
\hline \multicolumn{5}{|c|}{ Bonos en pesos } & \multicolumn{4}{c|}{ Bonos en dólares } \\
\hline Sticker & TIR & DM & TIR+CER & Sticker & TIR & DM \\
& & & & & $48,29 \%$ & 2,84 \\
TC21 & $10,58 \%$ & 1,02 & $57,30 \%$ & AA26D & $33,12 \%$ & 3,27 \\
PR13 & $10,18 \%$ & 1,78 & $51,98 \%$ & DICAD ley Argentina Canje 2005 & $3,19 \%$ & 3,21 \\
TC23 & $12,55 \%$ & 2,4 & $46,65 \%$ & DICO ley Argentina Canje 2010 & $34,19 \%$ \\
DICP & $12,11 \%$ & 5,62 & $32,11 \%$ & AA46 D & $27,42 \%$ & 3,86 \\
CUAP & $10,97 \%$ & 10,1 & $25,97 \%$ & PARD ley Argentina Canje 2010 & $17,95 \%$ & 7,12 \\
\hline
\end{tabular}

Fuente: elaboración propia en base a IAMC.

Gráfico A 2.1: Evolución del EMAE general 2019-2004

EMAE General (2004-2019)

$12,000000 \%$

$10,000000 \%$

$8,000000 \%$

$6,000000 \%$

$4,000000 \%$

$2,000000 \%$

$0,000000 \%$

$-2,000000 \%$

$-4,000000 \%$

$-6,000000 \%$

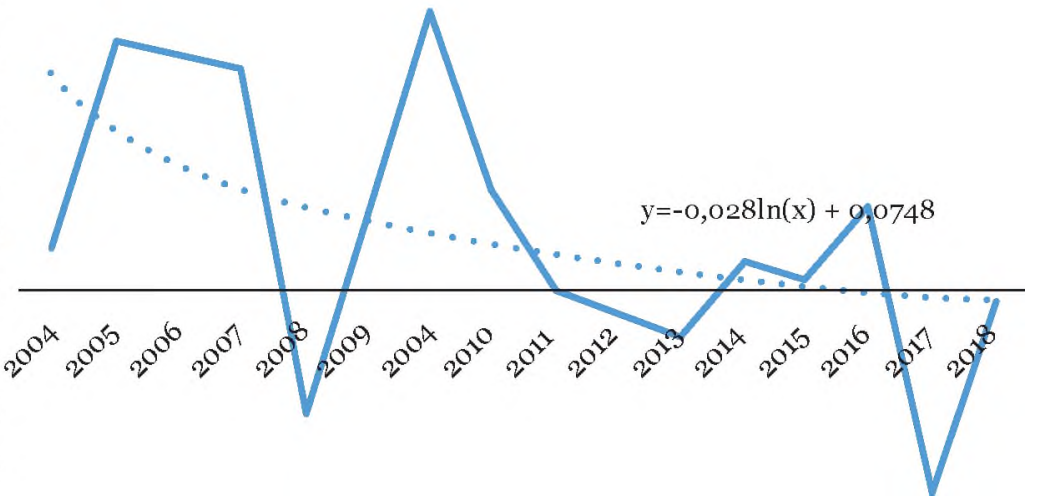

$-8,000000 \%$

Fuente: elaboración propia.

Gráfico A 2.2: Evolución Inflación en EE.UU 2019-2011 Inflación EE.UU

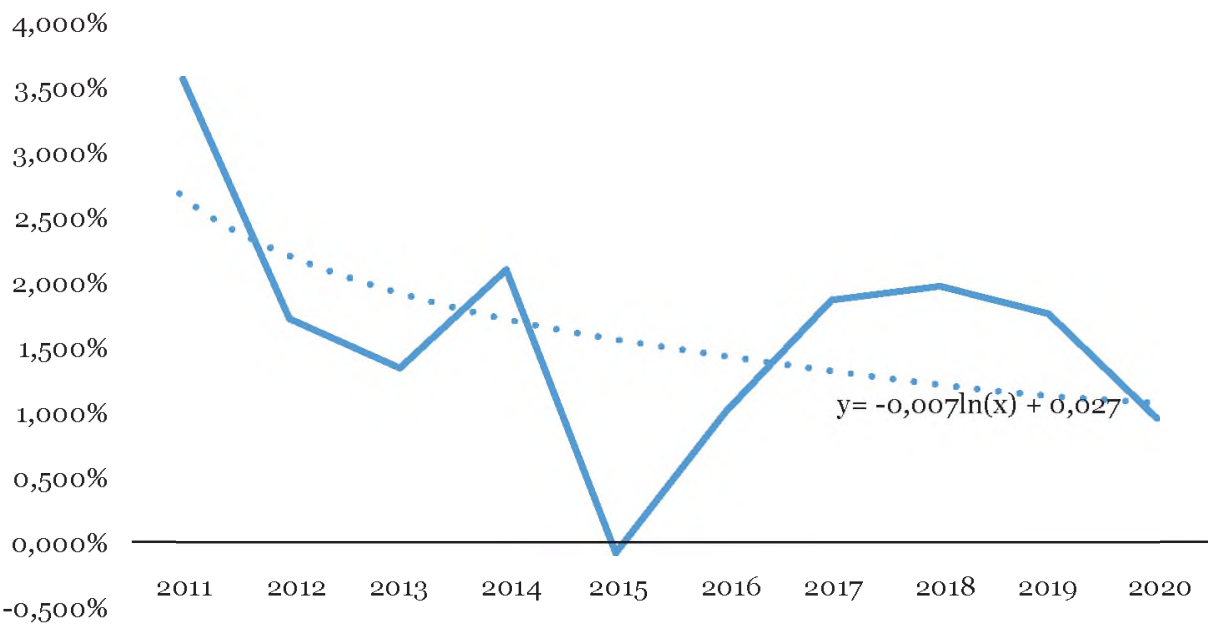

Fuente: elaboración propia. 
Gráfico A 2.3: Curva de rendimientos bonos soberanos en pesos (tabla A.2.1)

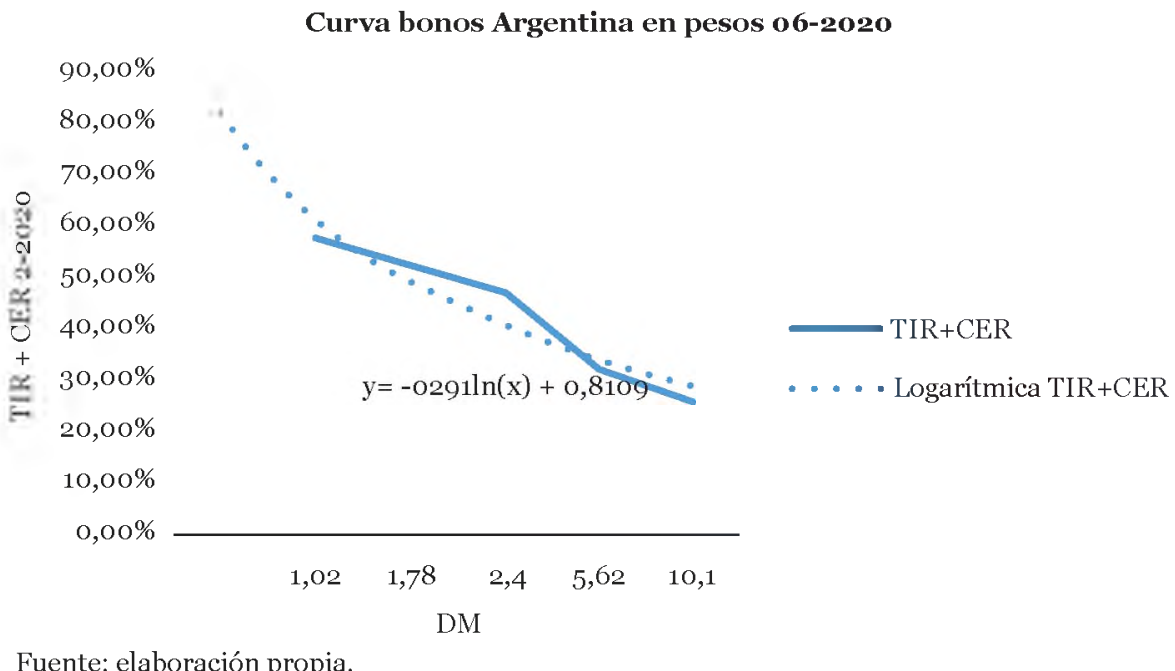

Gráfico A 2.4: Curva de rendimientos bonos soberanos en dólares EE.UU (tabla A.2.1)

Curva bonos Argentina en dólares o6-2020

0,8

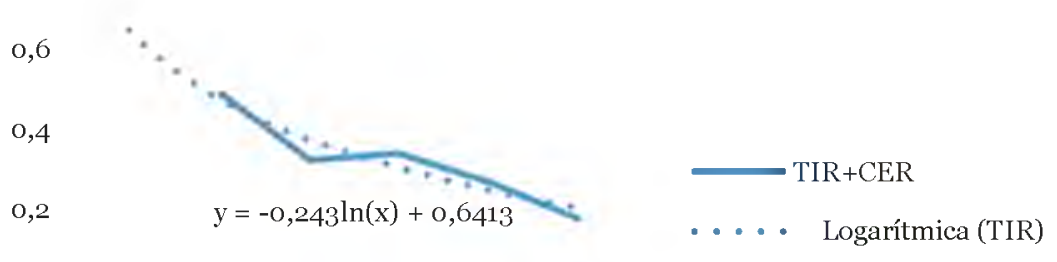

$2,84 \quad 3,27 \quad 3,21 \quad 3,86 \quad 7,12$

DM

Fuente: elaboración propia. 